\title{
Convolution identities for Tetranacci numbers
}

\author{
Takao Komatsu ${ }^{1}$ and Rusen $\mathbf{L i}^{2}$ \\ ${ }^{1}$ Department of Mathematical Sciences, School of Science \\ Zhejiang Sci-Tech University \\ Hangzhou, 310018, P. R. China \\ e-mail: komatsulzstu.edu.cn \\ ${ }^{2}$ School of Mathematics, Shandong University \\ Jinan, 250100, P. R. China \\ e-mail: limanjiashe@163.com
}

Received: 11 January 2019

Accepted: 14 July 2019

\begin{abstract}
Convolution identities for various numbers (e.g., Bernoulli, Euler, Genocchi, Catalan, Cauchy and Stirling numbers) have been studied by many authors. Recently, several convolution identities have been studied for Fibonacci and Tribonacci numbers too. In this paper, we give convolution identities with and without binomial (multinomial) coefficients for Tetranacci numbers, and convolution identities with binomial coefficients for Tetranacci and Tetranacci-type numbers.
\end{abstract} Keywords: Tetranacci numbers, Convolutions, Symmetric formulae.

2010 Mathematics Subject Classification: 11B39, 11B37, 05A15, 05A19.

\section{Introduction}

Convolution identities (or the sums of products) for various types of numbers (or polynomials) have been studied, with or without binomial coefficients, including the Bernoulli, Euler, Genocchi, Catalan, Cauchy and Stirling numbers (see, e.g., $[1-3,5,6,9,10]$ and the references therein). One typical well-known formula is due to Euler, given by

$$
\sum_{k=0}^{n}\left(\begin{array}{l}
n \\
k
\end{array}\right) \mathcal{B}_{k} \mathcal{B}_{n-k}=-n \mathcal{B}_{n-1}-(n-1) \mathcal{B}_{n} \quad(n \geq 0),
$$

where $\mathcal{B}_{n}$ are the Bernoulli numbers, defined by

$$
\frac{x}{e^{x}-1}=\sum_{n=0}^{\infty} \mathcal{B}_{n} \frac{x^{n}}{n !} \quad(|x|<2 \pi) \text {. }
$$


Some convolution identities for the Fibonacci numbers $F_{n}$ were established by Komatsu, Masakova and Pelantova [9]. Recently, several convolution identities with and without binomial coefficients for Tribonacci numbers $T_{n}$ were obtained by Komatsu [7]. By using symmetric formulae, more various identities for Tribonacci and Tribonacci-type numbers were given by Komatsu and Li [8].

The Tetranacci numbers $\mathfrak{T}_{n}$ are defined by

$$
\mathfrak{T}_{n}=\mathfrak{T}_{n-1}+\mathfrak{T}_{n-2}+\mathfrak{T}_{n-3}+\mathfrak{T}_{n-4} \quad(n \geq 4) \quad \text { with } \quad \mathfrak{T}_{0}=0, \mathfrak{T}_{1}=\mathfrak{T}_{2}=1, \mathfrak{T}_{3}=2
$$

and their sequence is given by

$$
\left\{\mathfrak{T}_{n}\right\}_{n \geq 0}=0,1,1,2,4,8,15,29,56,108,208, \ldots
$$

([12, A000078]).

The generating function without factorials is given by

$$
\mathfrak{T}(x):=\frac{x}{1-x-x^{2}-x^{3}-x^{4}}=\sum_{n=0}^{\infty} \mathfrak{T}_{n} x^{n}
$$

because of the recurrence relation (1).

On the other hand, the generating function with binomial coefficients is given by

$$
\mathfrak{t}(x):=c_{1} e^{\alpha x}+c_{2} e^{\beta x}+c_{3} e^{\gamma x}+c_{4} e^{\delta x}=\sum_{n=0}^{\infty} \mathfrak{T}_{n} \frac{x^{n}}{n !},
$$

where $\alpha, \beta, \gamma$ and $\delta$ are the roots of $x^{4}-x^{3}-x^{2}-x-1=0$ and

$$
\begin{aligned}
c_{1}: & =\frac{2-(\beta+\gamma+\delta)+(\beta \gamma+\gamma \delta+\delta \beta)}{(\alpha-\beta)(\alpha-\gamma)(\alpha-\delta)} \\
& =\frac{1}{-\alpha^{3}+6 \alpha-1}, \\
c_{2}: & =\frac{2-(\alpha+\gamma+\delta)+(\alpha \gamma+\gamma \delta+\delta \alpha)}{(\beta-\alpha)(\beta-\gamma)(\beta-\delta)} \\
& =\frac{1}{-\beta^{3}+6 \beta-1}, \\
c_{3}: & =\frac{2-(\alpha+\beta+\delta)+(\alpha \beta+\beta \delta+\delta \alpha)}{(\gamma-\alpha)(\gamma-\beta)(\gamma-\delta)} \\
& =\frac{1}{-\gamma^{3}+6 \gamma-1}, \\
c_{4}: & =\frac{2-(\alpha+\beta+\gamma)+(\alpha \beta+\beta \gamma+\gamma \alpha)}{(\delta-\alpha)(\delta-\beta)(\delta-\gamma)} \\
& =\frac{1}{-\delta^{3}+6 \delta-1} .
\end{aligned}
$$

Notice that

$$
\begin{aligned}
c_{1}+c_{2}+c_{3}+c_{4} & =0, \\
c_{1} \alpha+c_{2} \beta+c_{3} \gamma+c_{4} \delta & =1, \\
c_{1} \alpha^{2}+c_{2} \beta^{2}+c_{3} \gamma^{2}+c_{4} \delta^{2} & =1, \\
c_{1} \alpha^{3}+c_{2} \beta^{3}+c_{3} \gamma^{3}+c_{4} \delta^{3} & =2,
\end{aligned}
$$


because $\mathfrak{T}_{n}$ has a Binet-type formula:

$$
\mathfrak{T}_{n}=c_{1} \alpha^{n}+c_{2} \beta^{n}+c_{3} \gamma^{n}+c_{4} \gamma^{n} \quad(n \geq 0) .
$$

There have not been many papers about the Tetranacci numbers, though they are a special case of the so-called Fibonacci $s$-step numbers or $s$-generalized Fibonacci numbers $F_{n}^{(s)}$, defined by

$$
F_{n}^{(s)}=F_{n-1}^{(s)}+F_{n-2}^{(s)}+\cdots+F_{n-s}^{(s)}
$$

with some initial values. Clearly, for $s=4$, we have the Tetranacci numbers $\mathfrak{T}_{n}=F_{n}^{(4)}$.

De Moivre-type identities for the Tetranacci numbers ${ }^{1}$ and several related identities were given by Lin [11]. Some properties of the Tetranacci sequence modulo $m$ and certain identities involving them were established by Waddill $[13,14]$. Some congruences for lacunary sequences were derived as an analogous result for the Tetranacci numbers by Young [15]. By using matrix methods, explicit formulas for the Tribonacci and Tetranacci numbers were given by Kiric [4].

In this paper, we give convolution identities with and without binomial (multinomial) coefficients for the Tetranacci numbers and convolution identities with binomial coefficients for the Tetranacci and Tetranacci-type numbers. Some results are based upon symmetric formulae.

It may be possible to consider convolution identities for more general, Fibonacci $s$-step numbers, including pentanacci numbers $(s=5,[12$, A001591]), hexanacci numbers $(s=6,[12$, A001592]) and heptanacci numbers ( $s=7$, [12, A066178]). However, when $s \geq 5$, by the AbelRuffini theorem (in 1799, 1824), there is no algebraic expression for general quintic equations over the rationals in terms of radicals. This result also holds for equations of higher degrees. Therefore, to consider the identities exactly for the Tetranacci numbers would be meaningful.

\section{Convolution identities without binomial coefficients}

By (2), we have

$$
\mathfrak{T}^{\prime}(x)=\frac{1+x^{2}+2 x^{3}+3 x^{4}}{\left(1-x-x^{2}-x^{3}-x^{4}\right)^{2}}
$$

Hence,

$$
\left(1+x^{2}+2 x^{3}+3 x^{4}\right) \mathfrak{T}(x)^{2}=x^{2} \mathfrak{T}^{\prime}(x) .
$$

The left-hand side of (4) is

$$
\begin{aligned}
& \left(1+x^{2}+2 x^{3}+3 x^{4}\right) \sum_{n=0}^{\infty} \sum_{k=0}^{n} \mathfrak{T}_{k} \mathfrak{T}_{n-k} x^{n} \\
& =\sum_{n=0}^{\infty} \sum_{k=0}^{n} \mathfrak{T}_{k} \mathfrak{T}_{n-k} x^{n}+\sum_{n=2}^{\infty} \sum_{k=0}^{n-2} \mathfrak{T}_{k} \mathfrak{T}_{n-k-2} x^{n} \\
& \quad+2 \sum_{n=3}^{\infty} \sum_{k=0}^{n-3} \mathfrak{T}_{k} \mathfrak{T}_{n-k-3} x^{n}+3 \sum_{n=4}^{\infty} \sum_{k=0}^{n-4} \mathfrak{T}_{k} \mathfrak{T}_{n-k-4} x^{n} \\
& =\sum_{n=4}^{\infty} \sum_{k=0}^{n-4} \mathfrak{T}_{k}\left(\mathfrak{T}_{n-k}+\mathfrak{T}_{n-k-2}+2 \mathfrak{T}_{n-k-3}+3 \mathfrak{T}_{n-k-4}\right) x^{n}
\end{aligned}
$$

\footnotetext{
${ }^{1}$ In [11], it is called Tetrabonacci numbers.
} 


$$
+\sum_{n=4}^{\infty}\left(\mathfrak{T}_{n-1}+\mathfrak{T}_{n-2}+3 \mathfrak{T}_{n-3}\right) x^{n}+x^{2}+2 x^{3} .
$$

The right-hand side of (4) is

$$
x^{2} \sum_{n=0}^{\infty}(n+1) \mathfrak{T}_{n+1} x^{n}=\sum_{n=2}^{\infty}(n-1) \mathfrak{T}_{n-1} x^{n} .
$$

Therefore, we get the following result.

Theorem 2.1. For $n \geq 4$, we have

$$
\sum_{k=0}^{n-4} \mathfrak{T}_{k}\left(\mathfrak{T}_{n-k}+\mathfrak{T}_{n-k-2}+2 \mathfrak{T}_{n-k-3}+3 \mathfrak{T}_{n-k-4}\right)=(n-2) \mathfrak{T}_{n-1}-\mathfrak{T}_{n-2}-3 \mathfrak{T}_{n-3} .
$$

The identity (4) can be written as

$$
\mathfrak{T}(x)^{2}=\frac{x^{2}}{1+x^{2}+2 x^{3}+3 x^{4}} \mathfrak{T}^{\prime}(x) .
$$

Since

$$
\begin{aligned}
\frac{1}{1+x^{2}+2 x^{3}+3 x^{4}}= & \sum_{l=0}^{\infty}(-1)^{l} x^{2 l}\left(1+2 x+3 x^{2}\right)^{l} \\
= & \sum_{l=0}^{\infty}(-1)^{l} x^{2 l} \sum_{\substack{i+j+k=l \\
i, j, k \geq 0}}\left(\begin{array}{c}
l \\
i, j, k
\end{array}\right) 1^{i}(2 x)^{j}\left(3 x^{2}\right)^{k} \\
= & \sum_{m=0}^{\infty} \sum_{j, k=0}^{\substack{j j+2 k \leq m \\
j+4 k \leq m}}(-1)^{\frac{m-j-2 k}{2}} \frac{1+(-1)^{m-j-2 k}}{2} \\
& \times\left(\begin{array}{c}
\frac{m-j-2 k}{2} \\
\frac{m-2 k}{2}-j-k, j, k
\end{array}\right) 2^{j} 3^{k} x^{m},
\end{aligned}
$$

and

$$
\mathfrak{T}^{\prime}(x)=\sum_{n=0}^{\infty}(n+1) \mathfrak{T}_{n+1} x^{n}
$$

the right-hand side of (5) is

$$
\begin{aligned}
x^{2} A \sum_{l=0}^{\infty}(l+1) \mathfrak{T}_{l+1} x^{l} & =x^{2} \sum_{n=0}^{\infty} \sum_{l=0}^{n} B(l+1) \mathfrak{T}_{l+1} x^{n} \\
& =\sum_{n=2}^{\infty} \sum_{l=0}^{n-2} C(l+1) \mathfrak{T}_{l+1} x^{n},
\end{aligned}
$$

where

$$
A=\sum_{m=0}^{\infty} \sum_{j, k=0}^{\substack{3 j+2 k \leq m \\
j+4 k \leq m}}(-1)^{\frac{m-j-2 k}{2}} \frac{1+(-1)^{m-j-2 k}}{2}\left(\begin{array}{c}
\frac{m-j-2 k}{2} \\
\frac{m-j-2 k}{2}-j-k, j, k
\end{array}\right) 2^{j} 3^{k} x^{m}
$$




$$
\begin{aligned}
& B=\sum_{\substack{j, k=0 \\
3 j+2 k \leq n-2-l \\
j+4 k \leq n-2-l}}^{\substack{3 j+2 k \leq n-l \\
j+4 k \leq n-l}}(-1)^{\frac{n-l-j-2 k}{2}} \frac{1+(-1)^{n-l-j-2 k}}{2}\left(\begin{array}{c}
\frac{n-l-j-2 k}{2} \\
\frac{n-l-3 j-4 k}{2}, j, k
\end{array}\right) 2^{j} 3^{k}, \\
& C=\sum_{j, k=0}^{\frac{n-2-l-j-2 k}{2}} \frac{1+(-1)^{n-2-l-j-2 k}}{2}\left(\begin{array}{c}
\frac{n-2-l-j-2 k}{2} \\
\frac{n-2-l-3 j-4 k}{2}, j, k
\end{array}\right) 2^{j} 3^{k} .
\end{aligned}
$$

Since the left-hand side of (5) is

$$
\sum_{n=0}^{\infty} \sum_{k=0}^{n} \mathfrak{T}_{k} \mathfrak{T}_{n-k} x^{n}
$$

comparing the coefficients on both sides, we obtain the following result without binomial coefficient.

Theorem 2.2. For $n \geq 2$,

$$
\sum_{k=0}^{n} \mathfrak{T}_{k} \mathfrak{T}_{n-k}=\sum_{l=0}^{n-2}(l+1) \mathfrak{T}_{l+1} D
$$

where

$$
D=\sum_{j, k=0}^{\substack{3 j+2 k \leq n-2-l \\
j+4 k \leq n-2-l}}(-1)^{\frac{n-2-l-j-2 k}{2}} \frac{1+(-1)^{n-2-l-j-2 k}}{2}\left(\begin{array}{c}
\frac{n-2-l-j-2 k}{2} \\
\frac{n-2-l-3 j-4 k}{2}, j, k
\end{array}\right) 2^{j} 3^{k} .
$$

\section{Some preliminary lemmas}

For convenience, we shall introduce modified Tetranacci numbers $\mathfrak{T}_{n}^{\left(s_{0}, s_{1}, s_{2}, s_{3}\right)}$, satisfying the recurrence relation

$$
\mathfrak{T}_{n}^{\left(s_{0}, s_{1}, s_{2}, s_{3}\right)}=\mathfrak{T}_{n-1}^{\left(s_{0}, s_{1}, s_{2}, s_{3}\right)}+\mathfrak{T}_{n-2}^{\left(s_{0}, s_{1}, s_{2}, s_{3}\right)}+\mathfrak{T}_{n-3}^{\left(s_{0}, s_{1}, s_{2}, s_{3}\right)}+\mathfrak{T}_{n-4}^{\left(s_{0}, s_{1}, s_{2}, s_{3}\right)} \quad(n \geq 4)
$$

with given initial values $\mathfrak{T}_{0}^{\left(s_{0}, s_{1}, s_{2}, s_{3}\right)}=s_{0}, \mathfrak{T}_{1}^{\left(s_{0}, s_{1}, s_{2}, s_{3}\right)}=s_{1}, \mathfrak{T}_{2}^{\left(s_{0}, s_{1}, s_{2}, s_{3}\right)}=s_{2}$, and $\mathfrak{T}_{3}^{\left(s_{0}, s_{1}, s_{2}, s_{3}\right)}=$ $s_{3}$. Hence, $\mathfrak{T}_{n}=\mathfrak{T}_{n}^{(0,1,1,2)}$ are the ordinary Tetranacci numbers.

First, we shall prove the following four lemmata.

Lemma 3.1. We have

$$
c_{1}^{2} e^{\alpha x}+c_{2}^{2} e^{\beta x}+c_{3}^{2} e^{\gamma x}+c_{4}^{2} e^{\delta x}=\frac{1}{563} \sum_{n=0}^{\infty} \mathfrak{T}_{n}^{(40,64,215,344)} \frac{x^{n}}{n !} .
$$

Proof. For Tetranacci-type numbers $s_{n}$, satisfying the recurrence relation $s_{n}=s_{n-1}+s_{n-2}+$ $s_{n-3}+s_{n-4}(n \geq 4)$ with given initial values $s_{0}, s_{1}, s_{2}$ and $s_{3}$, we have

$$
d_{1} e^{\alpha x}+d_{2} e^{\beta x}+d_{3} e^{\gamma x}+d_{4} e^{\delta x}=\sum_{n=0}^{\infty} s_{n} \frac{x^{n}}{n !} .
$$


Since $d_{1}, d_{2}, d_{3}$ and $d_{4}$ satisfy the system of the equations

$$
\begin{aligned}
d_{1}+d_{2}+d_{3}+d_{4} & =s_{0}, \\
d_{1} \alpha+d_{2} \beta+d_{3} \gamma+d_{4} \gamma & =s_{1}, \\
d_{1} \alpha^{2}+d_{2} \beta^{2}+d_{3} \gamma^{2}+d_{4} \gamma^{2} & =s_{2}, \\
d_{1} \alpha^{3}+d_{2} \beta^{3}+d_{3} \gamma^{3}+d_{4} \gamma^{3} & =s_{3},
\end{aligned}
$$

we have

$$
\begin{aligned}
& d_{1}=\frac{\left|\begin{array}{cccc}
s_{0} & 1 & 1 & 1 \\
s_{1} & \beta & \gamma & \delta \\
s_{2} & \beta^{2} & \gamma^{2} & \delta^{2} \\
s_{3} & \beta^{3} & \gamma^{3} & \delta^{3}
\end{array}\right|}{\left|\begin{array}{cccc}
1 & 1 & 1 & 1 \\
\alpha & \beta & \gamma & \delta \\
\alpha^{2} & \beta^{2} & \gamma^{2} & \delta^{2} \\
\alpha^{3} & \beta^{3} & \gamma^{3} & \delta^{3}
\end{array}\right|}=\frac{s_{0} \beta \gamma \delta+s_{2}(\beta+\gamma+\delta)-s_{3}-s_{1}(\beta \gamma+\beta \delta+\gamma \delta)}{(\beta-\alpha)(\gamma-\alpha)(\delta-\alpha)} \\
& d_{2}=\frac{\left|\begin{array}{cccc}
1 & s_{0} & 1 & 1 \\
\alpha & s_{1} & \gamma & \delta \\
\alpha^{2} & s_{2} & \gamma^{2} & \delta^{2} \\
\alpha^{3} & s_{3} & \gamma^{3} & \delta^{3}
\end{array}\right|}{\left|\begin{array}{cccc}
1 & 1 & 1 & 1 \\
\alpha & \beta & \gamma & \delta \\
\alpha^{2} & \beta^{2} & \gamma^{2} & \delta^{2} \\
\alpha^{3} & \beta^{3} & \gamma^{3} & \delta^{3}
\end{array}\right|}=\frac{s_{0} \gamma \delta \alpha+s_{2}(\gamma+\delta+\alpha)-s_{3}-s_{1}(\gamma \delta+\gamma \alpha+\delta \alpha)}{(\gamma-\beta)(\delta-\beta)(\alpha-\beta)} \\
& d_{3}=\frac{\left|\begin{array}{cccc}
1 & 1 & s_{0} & 1 \\
\alpha & \beta & s_{1} & \delta \\
\alpha^{2} & \beta^{2} & s_{2} & \delta^{2} \\
\alpha^{3} & \beta^{3} & s_{3} & \delta^{3}
\end{array}\right|}{\left|\begin{array}{cccc}
1 & 1 & 1 & 1 \\
\alpha & \beta & \gamma & \delta \\
\alpha^{2} & \beta^{2} & \gamma^{2} & \delta^{2} \\
\alpha^{3} & \beta^{3} & \gamma^{3} & \delta^{3}
\end{array}\right|}=\frac{s_{0} \delta \alpha \beta+s_{2}(\delta+\alpha+\beta)-s_{3}-s_{1}(\delta \alpha+\delta \beta+\alpha \beta)}{(\delta-\gamma)(\alpha-\gamma)(\beta-\gamma)},
\end{aligned}
$$




$$
d_{4}=\frac{\left|\begin{array}{cccc}
1 & 1 & 1 & s_{0} \\
\alpha & \beta & \gamma & s_{1} \\
\alpha^{2} & \beta^{2} & \gamma^{2} & s_{2} \\
\alpha^{3} & \beta^{3} & \gamma^{3} & s_{3}
\end{array}\right|}{\left|\begin{array}{cccc}
1 & 1 & 1 & 1 \\
\alpha & \beta & \gamma & \delta \\
\alpha^{2} & \beta^{2} & \gamma^{2} & \delta^{2} \\
\alpha^{3} & \beta^{3} & \gamma^{3} & \delta^{3}
\end{array}\right|}=\frac{s_{0} \alpha \beta \gamma+s_{2}(\alpha+\beta+\gamma)-s_{3}-s_{1}(\alpha \beta+\alpha \gamma+\beta \gamma)}{(\alpha-\delta)(\beta-\delta)(\gamma-\delta)}
$$

When $s_{0}=40, s_{1}=64, s_{2}=215$ and $s_{3}=344$, by $\alpha+\beta+\gamma+\delta=1, \beta \gamma+\beta \delta+\gamma \delta=$ $-1-(\alpha \beta+\alpha \gamma+\alpha \delta)=\alpha^{2}-\alpha-1, \alpha \beta \gamma \delta=1$ and $\alpha^{4}=\alpha^{3}+\alpha^{2}+\alpha+1$, we have

$$
d_{1}=\frac{40 \beta \gamma \delta+215(\beta+\gamma+\delta)-344-64(\beta \gamma+\beta \delta+\gamma \delta)}{(\beta-\alpha)(\gamma-\alpha)(\delta-\alpha)}=563 c_{1}^{2} .
$$

Similarly, we have $d_{2}=563 c_{2}^{2}, d_{3}=563 c_{3}^{2}$ and $d_{4}=563 c_{4}^{2}$.

\section{Lemma 3.2. We have}

$$
\begin{aligned}
\sum_{n=0}^{\infty} t_{n} \frac{x^{n}}{n !}= & c_{1} c_{2} e^{(\alpha+\beta) x}+c_{1} c_{3} e^{(\alpha+\gamma) x}+c_{1} c_{4} e^{(\alpha+\delta) x} \\
& +c_{2} c_{3} e^{(\beta+\gamma) x}+c_{2} c_{4} e^{(\beta+\delta) x}+c_{3} c_{4} e^{(\gamma+\delta) x},
\end{aligned}
$$

where

$$
t_{n}=\frac{1}{2}\left(\sum_{k=0}^{n}\left(\begin{array}{l}
n \\
k
\end{array}\right) \mathfrak{T}_{k} \mathfrak{T}_{n-k}-\frac{2^{n}}{563} \mathfrak{T}_{n}^{(40,64,215,344)}\right)
$$

Proof. Since

$$
\begin{aligned}
& \left(c_{1} e^{\alpha x}+c_{2} e^{\beta x}+c_{3} e^{\gamma x}+c_{4} e^{\delta x}\right)^{2} \\
& =c_{1}^{2} e^{\alpha x}+c_{2}^{2} e^{\beta x}+c_{3}^{2} e^{\gamma x}+c_{4}^{2} e^{\delta x}+2\left(c_{1} c_{2} e^{(\alpha+\beta) x}+c_{1} c_{3} e^{(\alpha+\gamma) x}+c_{1} c_{4} e^{(\alpha+\delta) x}\right. \\
& \left.\quad+c_{2} c_{3} e^{(\beta+\gamma) x}+c_{2} c_{4} e^{(\beta+\delta) x}+c_{3} c_{4} e^{(\gamma+\delta) x}\right),
\end{aligned}
$$

we can obtain the following identity:

$$
\begin{aligned}
\left(\sum_{n=0}^{\infty} \mathfrak{T}_{n} \frac{x^{n}}{n !}\right)^{2} & =\sum_{n=0}^{\infty} \sum_{k=0}^{n}\left(\begin{array}{l}
n \\
k
\end{array}\right) \mathfrak{T}_{k} \mathfrak{T}_{n-k} \frac{x^{n}}{n !} \\
& =\frac{1}{563} \sum_{n=0}^{\infty} \mathfrak{T}_{n}^{(40,64,215,344)} \frac{(2 x)^{n}}{n !}+2 \sum_{n=0}^{\infty} t_{n} \frac{x^{n}}{n !}
\end{aligned}
$$

Comparing the coefficients on both sides, we get the desired result. 
Lemma 3.3. We have

$$
c_{2} c_{3} c_{4} e^{\alpha x}+c_{3} c_{4} c_{1} e^{\beta x}+c_{4} c_{1} c_{2} e^{\gamma x}+c_{1} c_{2} c_{3} e^{\delta x}=-\frac{1}{563} \sum_{n=0}^{\infty} \mathfrak{T}_{n}^{(-5,2,13,32)} \frac{x^{n}}{n !} .
$$

Proof. In the proof of Lemma 3.1, we put $s_{0}=-5, s_{1}=2, s_{2}=13$ and $s_{3}=32$, instead. We have

$$
d_{1}=\frac{-5 \beta \gamma \delta+13(\beta+\gamma+\delta)-32-2(\beta \gamma+\beta \delta+\gamma \delta)}{(\beta-\alpha)(\gamma-\alpha)(\delta-\alpha)}=-563 c_{2} c_{3} c_{4}
$$

Similarly, we have $d_{2}=-563 c_{3} c_{4} c_{1}, \quad d_{3}=-563 c_{4} c_{1} c_{2}$ and $d_{4}=-563 c_{1} c_{2} c_{3}$.

Lemma 3.4. We have

$$
c_{1} c_{2} c_{3} c_{4}=-\frac{1}{563}
$$

Proof. By $\alpha+\beta+\gamma+\delta=1, \beta \gamma+\beta \delta+\gamma \delta=-1-(\alpha \beta+\alpha \gamma+\alpha \delta)=\alpha^{2}-\alpha-1, \alpha \beta \gamma \delta=1$ and $\alpha^{4}=\alpha^{3}+\alpha^{2}+\alpha+1$, we have

$$
\begin{aligned}
c_{1} c_{2} c_{3} c_{4} & \frac{\alpha^{2}}{(\alpha-\beta)(\alpha-\gamma)(\alpha-\delta)} \frac{\beta^{2}}{(\beta-\alpha)(\beta-\gamma)(\beta-\delta)} \\
& \times \frac{\gamma^{2}}{(\gamma-\alpha)(\gamma-\beta)(\gamma-\delta)} \frac{\delta^{2}}{(\delta-\alpha)(\delta-\beta)(\delta-\gamma)} \\
= & \frac{\alpha^{2} \beta^{2} \gamma^{2} \delta^{2}}{(\alpha-\beta)^{2}(\alpha-\gamma)^{2}(\alpha-\delta)^{2}(\beta-\gamma)^{2}(\gamma-\beta)^{2}(\beta-\delta)^{2}} \\
= & \frac{1}{\left(4 \alpha^{3}-3 \alpha^{2}-2 \alpha-1\right)^{2}\left(39 \alpha^{3}-58 \alpha^{2}-23 \alpha-23\right)} \\
= & -\frac{1}{563} .
\end{aligned}
$$

\section{Convolution identities for three and four Tetranacci numbers}

Before giving more convolution identities, we shall give some elementary algebraic identities in symmetric form. It is not so difficult to determine the relations among coefficients.

Lemma 4.1. The following equality holds:

$$
\begin{aligned}
&(a+b+c+d)^{3} \\
&= A\left(a^{3}+b^{3}+c^{3}+d^{3}\right)+B(a b c+a b d+a c d+b c d) \\
&+C\left(a^{2}+b^{2}+c^{2}+d^{2}\right)(a+b+c+d) \\
&+D(a b+a c+a d+b c+b d+c d)(a+b+c+d),
\end{aligned}
$$

where $A=D-2, \quad B=-3 D+6, \quad C=-D+3$. 


\section{Lemma 4.2. The following equality holds:}

$$
\begin{aligned}
&(a+b+c+d)^{4} \\
&= A\left(a^{4}+b^{4}+c^{4}+d^{4}\right)+B a b c d+C\left(a^{3}+b^{3}+c^{3}+d^{4}\right)(a+b+c+d) \\
&+D\left(a^{2}+b^{2}+c^{2}+d^{2}\right)^{2}+E\left(a^{2}+b^{2}+c^{2}+d^{2}\right)(a b+a c+a d+b c+b d+c d) \\
&+F(a b+a c+a d+b c+b d+c d)^{2}+G\left(a^{2}+b^{2}+c^{2}+d^{2}\right)(a+b+c+d)^{2} \\
&+H(a b+a c+a d+b c+b d+c d)(a+b+c)^{2} \\
&+I(a b c(a+b+c)+a b d(a+b+d)+b c d(b+c+d)+a c d(a+c+d)) \\
&+J(a b c+a b d+b c d+a c d)(a+b+c+d),
\end{aligned}
$$

where $A=-D+E+G+H-3, \quad B=12 D+12 G-4 J-12, \quad C=-E-2 G-H+4$, $F=-2 D-2 G-2 H+6, \quad I=4 D-E+2 G-H-J$.

\section{Lemma 4.3. The following equality holds:}

$$
\begin{aligned}
(a+ & b+c+d)^{5} \\
= & A\left(a^{5}+b^{5}+c^{5}+d^{5}\right) \\
+ & B(a b c(a b+b c+c a)+a b d(a b+b d+a d)+a c d(a c+a d+c d)+b c d(b c+b d+c d)) \\
+ & C\left(a b c\left(a^{2}+b^{2}+c^{2}\right)+a b d\left(b^{2}+c^{2}+d^{2}\right)\right. \\
& \left.+a c d\left(a^{2}+c^{2}+d^{2}\right)+b c d\left(b^{2}+c^{2}+d^{2}\right)\right) \\
+ & D\left(a b c(a+b+c)^{2}+a b d(a+b+d)^{2}+a c d(a+c+d)^{2}+b c d(b+c+d)^{2}\right) \\
+ & E\left(a^{4}+b^{4}+c^{4}+d^{4}\right)(a+b+c+d)+F(a+b+c+d) a b c d \\
+ & G(a+b+c+d) \\
& \times(a b c(a+b+c)+a b d(a+b+d)+b c d(b+c+d)+a c d(a+c+d)) \\
+ & H\left(a^{3}+b^{3}+c^{3}+d^{3}\right)\left(a^{2}+b^{2}+c^{2}+d^{2}\right) \\
+ & I\left(a^{3}+b^{3}+c^{3}+d^{3}\right)(a b+a c+a d+b c+b d+c d) \\
+ & J(a b c+a b d+a c d+b c d)\left(a^{2}+b^{2}+c^{2}+d^{2}\right) \\
+ & K(a b c+a b d+a c d+b c d)(a b+a c+a d+b c+b d+c d) \\
+ & L\left(a^{3}+b^{3}+c^{3}+d^{3}\right)(a+b+c+d)^{2} \\
+ & M(a b c+a b d+a c d+b c d)(a+b+c+d)^{2} \\
+ & N\left(a^{2}+b^{2}+c^{2}+d^{2}\right)^{2}(a+b+c+d) \\
+ & P(a b+a c+a d+b c+b d+c d)^{2}(a+b+c+d) \\
+ & Q\left(a^{2}+b^{2}+c^{2}+d^{2}\right)(a b+a c+a d+b c+b d+c d)(a+b+c+d) \\
+ & R\left(a^{2}+b^{2}+c^{2}+d^{2}\right)(a+b+c+d)^{3} \\
+ & S(a b+a c+a d+b c+b d+c d)(a+b+c+d)^{3},
\end{aligned}
$$

where

$$
\begin{aligned}
& A=I+2 L+2 N+P+2 Q+6 R+4 S-14 \\
& B=-2 D-2 G-K-2 M-2 N-5 P-2 Q-6 R-12 S+30
\end{aligned}
$$




$$
\begin{aligned}
& C=-D-G-I-J-2 L-M-2 P-3 Q-6 R-7 S+20, \\
& E=-I-2 L-N-Q-3 R-S+5, \\
& F=-3 G-J-3 K-7 M-12 P-3 Q-6 R-27 S+60, \\
& H=-L-2 N-P-Q-4 R-3 S+10 .
\end{aligned}
$$

Now, let us consider the sum of three products with trinomial coefficients.

Lemma 4.4. We have

$$
c_{1}^{3} e^{\alpha x}+c_{2}^{3} e^{\beta x}+c_{3}^{3} e^{\gamma x}+c_{4}^{3} e^{\delta x}=\frac{1}{563} \sum_{n=0}^{\infty} \mathfrak{T}_{n}^{(15,27,48,107)} \frac{x^{n}}{n !} .
$$

Proof. In the proof of Lemma 3.1, we put $s_{0}=15, s_{1}=27, s_{2}=48$ and $s_{3}=107$, instead. We can obtain that

$$
d_{1}=\frac{15 \beta \gamma \delta+48(\beta+\gamma+\delta)-107-27(\beta \gamma+\beta \delta+\gamma \delta)}{(\beta-\alpha)(\gamma-\alpha)(\delta-\alpha)}=563 c_{1}^{3} .
$$

. Similarly, we have $d_{2}=563 c_{2}^{3}, d_{3}=563 c_{3}^{3}$ and $d_{4}=563 c_{4}^{3}$.

By using Lemmata 3.1, 3.2, 3.3, 4.1 and 4.4, we get the following result.

Theorem 4.5. For $n \geq 0$,

$$
\begin{aligned}
& \sum_{\substack{k_{1}+k_{2}+k_{3}=n \\
k_{1}, k_{2}, k_{3} \geq 0}}\left(\begin{array}{c}
n \\
k_{1}, k_{2}, k_{3}
\end{array}\right) \mathfrak{T}_{k_{1}} \mathfrak{T}_{k_{2}} \mathfrak{T}_{k_{3}} \\
= & \frac{A}{563} 3^{n} \mathfrak{T}_{n}^{(15,27,48,107)}-\frac{B}{563} \sum_{k=0}^{n}\left(\begin{array}{l}
n \\
k
\end{array}\right) \mathfrak{T}_{k}^{(-5,2,13,32)}(-1)^{k} \\
& +\frac{C}{563} \sum_{k=0}^{n}\left(\begin{array}{l}
n \\
k
\end{array}\right) 2^{n-k} \mathfrak{T}_{n-k}^{(40,64,215,344)} \mathfrak{T}_{k}+D \sum_{k=0}^{n}\left(\begin{array}{l}
n \\
k
\end{array}\right) \mathfrak{T}_{k} t_{n-k} .
\end{aligned}
$$

where $A=D-2, B=-3 D+6, C=-D+3$, and $t_{n}$ in given in Lemma 3.2 .

Remark. If we take $D=0$, we have for $n \geq 0$,

$$
\begin{aligned}
& \sum_{\substack{k_{1}+k_{2}+k_{3}=n \\
k_{1}, k_{2}, k_{3} \geq 0}}\left(\begin{array}{c}
n \\
k_{1}, k_{2}, k_{3}
\end{array}\right) \mathfrak{T}_{k_{1}} \mathfrak{T}_{k_{2}} \mathfrak{T}_{k_{3}} \\
= & -\frac{2}{563} 3^{n} \mathfrak{T}_{n}^{(15,27,48,107)}-\frac{6}{563} \sum_{k=0}^{n}\left(\begin{array}{l}
n \\
k
\end{array}\right) \mathfrak{T}_{k}^{(-5,2,13,32)}(-1)^{k} \\
& +\frac{3}{563} \sum_{k=0}^{n}\left(\begin{array}{l}
n \\
k
\end{array}\right) 2^{n-k} \mathfrak{T}_{n-k}^{(40,64,215,344)} \mathfrak{T}_{k} .
\end{aligned}
$$


Proof. First, by Lemmata 3.1, 3.2, 3.3, 4.1 and 4.4, we have

$$
\begin{aligned}
\left(c_{1} e^{\alpha x}+c_{2} e^{\beta x}+c_{3} e^{\gamma x}+c_{4} e^{\delta x}\right)^{3} \\
=A\left(c_{1}^{3} e^{3 \alpha x}+c_{2}^{3} e^{3 \beta x}+c_{3}^{3} e^{3 \gamma x}+c_{4}^{3} e^{3 \delta x}\right) \\
\quad+B\left(c_{1} c_{2} c_{3} e^{(\alpha+\beta+\gamma) x}+c_{2} c_{3} c_{4} e^{(\beta+\gamma+\delta) x}+c_{1} c_{2} c_{4} e^{(\alpha+\beta+\delta) x}+c_{1} c_{3} c_{4} e^{(\alpha+\gamma+\delta) x}\right) \\
\quad+C\left(c_{1}^{2} e^{2 \alpha x}+c_{2}^{2} e^{2 \beta x}+c_{3}^{2} e^{2 \gamma x}+c_{4}^{2} e^{2 \delta x}\right)\left(c_{1} e^{\alpha x}+c_{2} e^{\beta x}+c_{3} e^{\gamma x}+c_{4} e^{\delta x}\right) \\
\quad+D\left(c_{1} c_{2} e^{(\alpha+\beta) x}+c_{1} c_{3} e^{(\alpha+\gamma) x}+c_{1} c_{4} e^{(\alpha+\delta) x}+c_{2} c_{3} e^{(\beta+\gamma) x}+c_{2} c_{4} e^{(\beta+\delta) x}+c_{3} c_{4} e^{(\gamma+\delta) x}\right) \\
\quad \times\left(c_{1} e^{\alpha x}+c_{2} e^{\beta x}+c_{3} e^{\gamma x}+c_{4} e^{\delta x}\right) \\
=\frac{A}{563} \sum_{n=0}^{\infty} \mathfrak{T}_{n}^{(15,27,48,107)} \frac{(3 x)^{n}}{n !}-\frac{B}{563} \sum_{n=0}^{\infty} \sum_{k=0}^{n}\left(\begin{array}{l}
n \\
k
\end{array}\right) \mathfrak{T}_{k}^{(-5,2,13,32)}(-1)^{k} \frac{x^{n}}{n !} \\
\quad+\frac{C}{563} \sum_{n=0}^{\infty} \sum_{k=0}^{n}\left(\begin{array}{l}
n \\
k
\end{array}\right) 2^{n-k} \mathfrak{T}_{n-k}^{(40,64,215,344)} \mathfrak{T}_{k} \frac{x^{n}}{n !}+D \sum_{n=0}^{\infty} \sum_{k=0}^{n}\left(\begin{array}{l}
n \\
k
\end{array}\right) \mathfrak{T}_{k} t_{n-k} \frac{x^{n}}{n !} .
\end{aligned}
$$

On the other hand,

$$
\left(\sum_{n=0}^{\infty} \mathfrak{T}_{n} \frac{x^{n}}{n !}\right)^{3}=\sum_{\substack{k_{1}+k_{2}+k_{3}=n \\
k_{1}, k_{2}, k_{3} \geq 0}}\left(\begin{array}{c}
n \\
k_{1}, k_{2}, k_{3}
\end{array}\right) \mathfrak{T}_{k_{1}} \mathfrak{T}_{k_{2}} \mathfrak{T}_{k_{3}} \frac{x^{n}}{n !} .
$$

Comparing the coefficients on both sides, we get the desired result.

Next, we shall consider the sum of the products of four Tetranacci numbers. We need the following supplementary result. The proof is similar to that of Lemma 4.4 and, hence, omitted.

Lemma 4.6. We have

$$
c_{1}^{4} e^{\alpha x}+c_{2}^{4} e^{\beta x}+c_{3}^{4} e^{\gamma x}+c_{4}^{4} e^{\delta x}=\frac{1}{563^{2}} \sum_{n=0}^{\infty} \mathfrak{T}_{n}^{(3052,4658,8804,16451)} \frac{x^{n}}{n !} .
$$

By using Lemmata 3.1, 3.2, 3.3, 4.2, 4.4, and 4.6, letting $I=0$ in Lemma 4.2, comparing the coefficients on both sides, we can get the following theorem.

Theorem 4.7. For $n \geq 0$,

$$
\begin{aligned}
& \sum_{\substack{k_{1}+k_{2}+k_{3}+k_{4}=n \\
k_{1}, k_{2}, k_{3}, k_{4} \geq 0}}\left(\begin{array}{c}
n \\
k_{1}, k_{2}, k_{3}, k_{4}
\end{array}\right) \mathfrak{T}_{k_{1}} \mathfrak{T}_{k_{2}} \mathfrak{T}_{k_{3}} \mathfrak{T}_{k_{4}} \\
& =\frac{A}{563^{2}} 4^{n} \mathfrak{T}_{n}^{(3052,4658,8804,16451)}-\frac{B}{563}+\frac{C}{563} \sum_{k=0}^{n}\left(\begin{array}{l}
n \\
k
\end{array}\right) 3^{n-k} \mathfrak{T}_{n-k}^{(15,27,48,107)} \mathfrak{T}_{k} \\
& \quad+\frac{D}{563^{2}} \sum_{k=0}^{n}\left(\begin{array}{l}
n \\
k
\end{array}\right) 2^{n} \mathfrak{T}_{n-k}^{(40,64,215,344)} \mathfrak{T}_{k}^{(40,64,215,344)} \\
& +\frac{E}{563} \sum_{k=0}^{n}\left(\begin{array}{l}
n \\
k
\end{array}\right) 2^{n-k} \mathfrak{T}_{n-k}^{(40,64,215,344)} t_{k}+F \sum_{k=0}^{n}\left(\begin{array}{l}
n \\
k
\end{array}\right) t_{n-k} t_{k}
\end{aligned}
$$




$$
\begin{aligned}
& +\frac{G}{563} \sum_{\substack{k_{1}+k_{2}+k_{3}=n \\
k_{1}, k_{2}, k_{3} \geq 0}}\left(\begin{array}{c}
n \\
k_{1}, k_{2}, k_{3}
\end{array}\right) \mathfrak{T}_{k_{1}}^{(40,64,215,344)} 2^{k_{1}} \mathfrak{T}_{k_{2}} \mathfrak{T}_{k_{3}} \\
& +H \sum_{\substack{k_{1}+k_{2}+k_{3}=n \\
k_{1}, k_{2}, k_{3} \geq 0}}\left(\begin{array}{c}
n \\
k_{1}, k_{2}, k_{3}
\end{array}\right) t_{k_{1}} \mathfrak{T}_{k_{2}} \mathfrak{T}_{k_{3}} \\
& -\frac{J}{563} \sum_{\substack{k_{1}+k_{2}+k_{3}=n \\
k_{1}, k_{2}, k_{3} \geq 0}}\left(\begin{array}{c}
n \\
k_{1}, k_{2}, k_{3}
\end{array}\right) \mathfrak{T}_{k_{1}}^{(-5,2,13,32)}(-1)^{k_{1}} \mathfrak{T}_{k_{2}},
\end{aligned}
$$

where $A=-D+E+G+H-3, B=-4 D+4 E+4 G+4 H-12, C=-E-2 G-H+4$, $F=-2 D-2 G-2 H+6, J=4 D-E+2 G-H$, and $t_{n}$ in given in Lemma 3.2.

Remark. If $D=E=G=H=0$, then by $A=-3, B=-12, C=4, F=6$ and $J=0$, we have for $n \geq 0$,

$$
\begin{aligned}
& \sum_{\substack{k_{1}+k_{2}+k_{3}+k_{4}=n \\
k_{1}, k_{2}, k_{3}, k_{4} \geq 0}}\left(\begin{array}{c}
n \\
k_{1}, k_{2}, k_{3}, k_{4}
\end{array}\right) \mathfrak{T}_{k_{1}} \mathfrak{T}_{k_{2}} \mathfrak{T}_{k_{3}} \mathfrak{T}_{k_{4}} \\
= & -\frac{3}{563^{2}} 4^{n} \mathfrak{T}_{n}^{(3052,4658,8804,16451)}+\frac{12}{563}+\frac{4}{563} \sum_{k=0}^{n}\left(\begin{array}{l}
n \\
k
\end{array}\right) 3^{n-k} \mathfrak{T}_{n-k}^{(15,27,48,107)} \mathfrak{T}_{k} \\
& +6 \sum_{k=0}^{n}\left(\begin{array}{l}
n \\
k
\end{array}\right) t_{n-k} t_{k} .
\end{aligned}
$$

Let

$$
\begin{aligned}
& \sum_{n=0}^{\infty} t_{n}^{(1)} \frac{x^{n}}{n !} \\
& =c_{1} c_{2} c_{3} e^{(\alpha+\beta+\gamma) x}\left(c_{1} e^{\alpha x}+c_{2} e^{\beta x}+c_{3} e^{\gamma x}\right)+c_{2} c_{3} c_{4} e^{(\beta+\gamma+\delta) x}\left(c_{2} e^{\beta x}+c_{3} e^{\gamma x}+c_{4} e^{\delta x}\right) \\
& \quad+c_{1} c_{2} c_{4} e^{(\alpha+\beta+\delta) x}\left(c_{1} e^{\alpha x}+c_{2} e^{\beta x}+c_{4} e^{\delta x}\right)+c_{1} c_{3} c_{4} e^{(\alpha+\gamma+\delta) x}\left(c_{1} e^{\alpha x}+c_{3} e^{\gamma x}+c_{4} e^{\delta x}\right) .
\end{aligned}
$$

By using Lemmata 3.1, 3.2, 3.3, 4.2, 4.4, and 4.6, comparing the coefficients on both sides, we can get the following theorem.

Theorem 4.8. For $n \geq 0, I \neq 0$

$$
\begin{aligned}
I t_{n}^{(1)}= & \sum_{\substack{k_{1}+k_{2}+k_{3}+k_{4}=n \\
k_{1}, k_{2}, k_{3}, k_{4} \geq 0}}\left(\begin{array}{c}
n \\
k_{1}, k_{2}, k_{3}, k_{4}
\end{array}\right) \mathfrak{T}_{k_{1}} \mathfrak{T}_{k_{2}} \mathfrak{T}_{k_{3}} \mathfrak{T}_{k_{4}} \\
& -\frac{A}{563^{2}} 4^{n} \mathfrak{T}_{n}^{(3052,4658,8804,16451)}+\frac{B}{563}-\frac{C}{563} \sum_{k=0}^{n}\left(\begin{array}{l}
n \\
k
\end{array}\right) 3^{n-k} \mathfrak{T}_{n-k}^{(15,27,48,107)} \mathfrak{T}_{k} \\
& -\frac{D}{563^{2}} \sum_{k=0}^{n}\left(\begin{array}{l}
n \\
k
\end{array}\right) 2^{n} \mathfrak{T}_{n-k}^{(40,64,215,344)} \mathfrak{T}_{k}^{(40,64,215,344)} \\
& -\frac{E}{563} \sum_{k=0}^{n}\left(\begin{array}{l}
n \\
k
\end{array}\right) 2^{n-k} \mathfrak{T}_{n-k}^{(40,64,215,344)} t_{k}-F \sum_{k=0}^{n}\left(\begin{array}{l}
n \\
k
\end{array}\right) t_{n-k} t_{k}
\end{aligned}
$$




$$
\begin{aligned}
& -\frac{G}{563} \sum_{\substack{k_{1}+k_{2}+k_{3}=n \\
k_{1}, k_{2}, k_{3} \geq 0}}\left(\begin{array}{c}
n \\
k_{1}, k_{2}, k_{3}
\end{array}\right) \mathfrak{T}_{k_{1}}^{(40,64,215,344)} 2^{k_{1}} \mathfrak{T}_{k_{2}} \mathfrak{T}_{k_{3}} \\
& -H \sum_{\substack{k_{1}+k_{2}+k_{3}=n \\
k_{1}, k_{2}, k_{3} \geq 0}}\left(\begin{array}{c}
n \\
k_{1}, k_{2}, k_{3}
\end{array}\right) t_{k_{1}} \mathfrak{T}_{k_{2}} \mathfrak{T}_{k_{3}} \\
& +\frac{J}{563} \sum_{\substack{k_{1}+k_{2}+k_{3}=n \\
k_{1}, k_{2}, k_{3} \geq 0}}\left(\begin{array}{c}
n \\
k_{1}, k_{2}, k_{3}
\end{array}\right) \mathfrak{T}_{k_{1}}^{(-5,2,13,32)}(-1)^{k_{1}} \mathfrak{T}_{k_{2}},
\end{aligned}
$$

where $A=-D+E+G+H-3, \quad B=12 D+12 G-4 J-12, \quad C=-E-2 G-H+4$, $F=-2 D-2 G-2 H+6, \quad I=4 D-E+2 G-H-J$, and $t_{n}$ is given in Lemma 3.2.

Remark. If $D=E=G=H=0, J=-1$, then by $A=-3, B=-8, C=4, F=6$ and $I=1$, we have for $n \geq 0$,

$$
\begin{aligned}
\mathfrak{T}_{n}^{(1)}= & \sum_{\substack{k_{1}+k_{2}+k_{3}+k_{4}=n \\
k_{1}, k_{2}, k_{3}, k_{4} \geq 0}}\left(\begin{array}{c}
n \\
k_{1}, k_{2}, k_{3}, k_{4}
\end{array}\right) \mathfrak{T}_{k_{1}} \mathfrak{T}_{k_{2}} \mathfrak{T}_{k_{3}} \mathfrak{T}_{k_{4}} \\
& +\frac{3}{563^{2}} 4^{n} \mathfrak{T}_{n}^{(3052,4658,8804,16451)}-\frac{8}{563}-\frac{4}{563} \sum_{k=0}^{n}\left(\begin{array}{l}
n \\
k
\end{array}\right) 3^{n-k} \mathfrak{T}_{n-k}^{(15,27,48,107)} \mathfrak{T}_{k} \\
& -6 \sum_{k=0}^{n}\left(\begin{array}{l}
n \\
k
\end{array}\right) t_{n-k} t_{k}-\frac{1}{563} \sum_{\substack{k_{1}+k_{2}+k_{3}=n \\
k_{1}, k_{2}, k_{3} \geq 0}}\left(\begin{array}{c}
n \\
k_{1}, k_{2}, k_{3}
\end{array}\right) \mathfrak{T}_{k_{1}}^{(-5,2,13,32)}(-1)^{k_{1}} \mathfrak{T}_{k_{2}} .
\end{aligned}
$$

\section{Convolution identities for five Tetranacci numbers}

We shall consider the sum of the products of five Tetranacci numbers. We need the following supplementary result. The proof is similar to that of Lemma 4.4 and, hence, omitted.

\section{Lemma 5.1.}

$$
c_{1}^{5} e^{\alpha x}+c_{2}^{5} e^{\beta x}+c_{3}^{5} e^{\gamma x}+c_{4}^{5} e^{\delta x}=\frac{1}{563^{2}} \sum_{n=0}^{\infty} \mathfrak{T}_{n}^{(500,1423,2598,4986)} \frac{x^{n}}{n !} .
$$

By using Lemmata 3.1, 3.2, 3.3, 4.3, 4.4, 4.6 and 5.1, comparing the coefficients on both sides, we can get the following theorems.

\subsection{Case 1}

Let $B=C=D=0$, we can obtain the following theorem.

Theorem 5.2. For $n \geq 0$,

$$
\sum_{\substack{k_{1}+\cdots+k_{5}=n \\
k_{1}, \ldots, k_{5} \geq 0}}\left(\begin{array}{c}
n \\
k_{1}, \ldots, k_{5}
\end{array}\right) \mathfrak{T}_{k_{1}} \cdots \mathfrak{T}_{k_{5}}
$$




$$
\begin{aligned}
& =\frac{A}{563^{2}} 5^{n} \mathfrak{T}_{n}^{(500,1423,2598,4986)}+\frac{E}{563^{2}} \sum_{k=0}^{n}\left(\begin{array}{l}
n \\
k
\end{array}\right) 4^{n-k} \mathfrak{T}_{n-k}^{(3052,4658,8804,16451)} \mathfrak{T}_{k} \\
& -\frac{F}{563} \sum_{k=0}^{n}\left(\begin{array}{l}
n \\
k
\end{array}\right) \mathfrak{T}_{k}+G \sum_{k=0}^{n}\left(\begin{array}{l}
n \\
k
\end{array}\right) t_{k}^{(1)} \mathfrak{T}_{n-k}+\frac{H}{563^{2}} \sum_{k=0}^{n}\left(\begin{array}{l}
n \\
k
\end{array}\right) 3^{n-k} 2^{k} \mathfrak{T}_{n-k}^{(15,27,48,107)} \mathfrak{T}_{k}^{(40,64,215,344)} \\
& +\frac{I}{563} \sum_{k=0}^{n}\left(\begin{array}{l}
n \\
k
\end{array}\right) 3^{k} \mathfrak{T}_{k}^{(15,27,48,107)} t_{n-k} \\
& -\frac{J}{563^{2}} \sum_{\substack{k_{1}+k_{2}+k_{3}=n \\
k_{1}, k_{2}, k_{3} \geq 0}}\left(\begin{array}{c}
n \\
k_{1}, k_{2}, k_{3}
\end{array}\right)(-1)^{k_{1}} 2^{k_{2}} \mathfrak{T}_{k_{1}}^{(-5,2,13,32)} \mathfrak{T}_{k_{2}}^{(40,64,215,344)} \\
& -\frac{K}{563} \sum_{\substack{k_{1}+k_{2}+k_{3}=n \\
k_{1}, k_{2}, k_{3} \geq 0}}\left(\begin{array}{c}
n \\
k_{1}, k_{2}, k_{3}
\end{array}\right)(-1)^{k_{1}} \mathfrak{T}_{k_{1}}^{(-5,2,13,32)} t_{k_{2}} \\
& +\frac{L}{563} \sum_{\substack{k_{1}+k_{2}+k_{3}=n \\
k_{1}, k_{2}, k_{3} \geq 0}}\left(\begin{array}{c}
n \\
k_{1}, k_{2}, k_{3}
\end{array}\right) 3^{k_{1}} \mathfrak{T}_{k_{1}}^{(15,27,48,107)} \mathfrak{T}_{k_{2}} \mathfrak{T}_{k_{3}} \\
& -\frac{M}{563} \sum_{\substack{k_{1}+k_{2}+k_{3}+k_{4}=n \\
k_{1}, k_{2}, k_{3}, k_{4} \geq 0}}\left(\begin{array}{c}
n \\
k_{1}, k_{2}, k_{3}, k_{4}
\end{array}\right)(-1)^{k_{1}} \mathfrak{T}_{k_{1}}^{(-5,2,13,32)} \mathfrak{T}_{k_{2}} \mathfrak{T}_{k_{3}} \\
& +\frac{N}{563^{2}} \sum_{\substack{k_{1}+k_{2}+k_{3}=n \\
k_{1}, k_{2}, k_{3} \geq 0}}\left(\begin{array}{c}
n \\
k_{1}, k_{2}, k_{3}
\end{array}\right) 2^{k_{1}} \mathfrak{T}_{k_{1}}^{(40,64,215,344)} 2^{k_{2}} \mathfrak{T}_{k_{2}}^{(40,64,215,344)} \mathfrak{T}_{k_{3}} \\
& +P \sum_{\substack{k_{1}+k_{2}+k_{3}=n \\
k_{1}, k_{2}, k_{3} \geq 0}}\left(\begin{array}{c}
n \\
k_{1}, k_{2}, k_{3}
\end{array}\right) t_{k_{1}} t_{k_{2}} \mathfrak{T}_{k_{3}} \\
& +\frac{Q}{563} \sum_{\substack{k_{1}+k_{2}+k_{3}=n \\
k_{1}, k_{2}, k_{3} \geq 0}}\left(\begin{array}{c}
n \\
k_{1}, k_{2}, k_{3}
\end{array}\right) 2^{k_{1}} \mathfrak{T}_{k_{1}}^{(40,64,215,344)} t_{k_{2}} \mathfrak{T}_{k_{3}} \\
& +\frac{R}{563} \sum_{\substack{k_{1}+k_{2}+k_{3}+k_{4}=n \\
k_{1}, k_{2}, k_{3}, k_{4} \geq 0}}\left(\begin{array}{c}
n \\
k_{1}, k_{2}, k_{3}, k_{4}
\end{array}\right) 2^{k_{1}} \mathfrak{T}_{k_{1}}^{(40,64,215,344)} \mathfrak{T}_{k_{2}} \mathfrak{T}_{k_{3}} \mathfrak{T}_{k_{4}} \\
& +S \sum_{\substack{k_{1}+k_{2}+k_{3}+k_{4}=n \\
k_{1}, k_{2}, k_{3}, k_{4} \geq 0}}\left(\begin{array}{c}
n \\
k_{1}, k_{2}, k_{3}, k_{4}
\end{array}\right) t_{k_{1}} \mathfrak{T}_{k_{2}} \mathfrak{T}_{k_{3}} \mathfrak{T}_{k_{4}}
\end{aligned}
$$

where

$$
\begin{aligned}
& A=I+2 L+2 N+P+2 Q+6 R+4 S-14, \\
& E=-I-2 L-N-Q-3 R-S+5, \\
& F=4 G+I+2 L+6 N+5 P+6 Q+18 R+16 S-50, \\
& H=-L-2 N-P-Q-4 R-3 S+10, \\
& J=-G-I-2 L-M-2 P-3 Q-6 R-7 S+20, \\
& K=-2 G-2 M-2 N-5 P-2 Q-6 R-12 S+30,
\end{aligned}
$$

$t_{n}$ and $t_{n}^{(1)}$ are same as those in Lemma 3.2 and Theorem 4.8, respectively. 
Remark. If $G=I=L=M=N=P=Q=R=S=0$, then by $A=-14, E=5$, $F=-50, H=10, J=20$ and $K=30$, we have for $n \geq 0$,

$$
\begin{aligned}
& \sum_{\substack{k_{1}+\ldots+k_{5}=n \\
k_{1}, \ldots, k_{5} \geq 0}}\left(\begin{array}{c}
n \\
k_{1}, \ldots, k_{5}
\end{array}\right) \mathfrak{T}_{k_{1}} \ldots \mathfrak{T}_{k_{5}} \\
= & -\frac{14}{563^{2}} 5^{n} \mathfrak{T}_{n}^{(500,1423,2598,4986)}+\frac{5}{563^{2}} \sum_{k=0}^{n}\left(\begin{array}{l}
n \\
k
\end{array}\right) 4^{n-k} \mathfrak{T}_{n-k}^{(3052,4658,8804,16451)} \mathfrak{T}_{k} \\
& +\frac{50}{563} \sum_{k=0}^{n}\left(\begin{array}{l}
n \\
k
\end{array}\right) \mathfrak{T}_{k}+\frac{10}{563^{2}} \sum_{k=0}^{n}\left(\begin{array}{l}
n \\
k
\end{array}\right) 3^{n-k} 2^{k} \mathfrak{T}_{n-k}^{(15,27,48,107)} \mathfrak{T}_{k}^{(40,64,215,344)} \\
& -\frac{20}{563^{2}} \sum_{\substack{k_{1}+k_{2}+k_{3}=n \\
k_{1}, k_{2}, k_{3} \geq 0}}\left(\begin{array}{c}
n \\
k_{1}, k_{2}, k_{3}
\end{array}\right)(-1)^{k_{1}} 2^{k_{2}} \mathfrak{T}_{k_{1}}^{(-5,2,13,32)} \mathfrak{T}_{k_{2}}^{(40,64,215,344)} \\
& -\frac{30}{563} \sum_{\substack{k_{1}+k_{2}+k_{3}=n \\
k_{1}, k_{2}, k_{3} \geq 0}}\left(\begin{array}{c}
n \\
k_{1}, k_{2}, k_{3}
\end{array}\right)(-1)^{k_{1}} \mathfrak{T}_{k_{1}}^{(-5,2,13,32)} t_{k_{2}} .
\end{aligned}
$$

\subsection{Case 2}

Let $B \neq 0, C=D=0$, we can obtain the following theorem.

Let

$$
\begin{aligned}
& \sum_{n=0}^{\infty} t_{n}^{(2)} \frac{x^{n}}{n !} \\
& =c_{1} c_{2} c_{3} e^{(\alpha+\beta+\gamma) x}\left(c_{1} c_{2} e^{(\alpha+\beta) x}+c_{2} c_{3} e^{(\beta+\gamma) x}+c_{3} c_{1} e^{(\gamma+\alpha) x}\right)+\cdots \\
& \quad+c_{2} c_{3} c_{4} e^{(\beta+\gamma+\delta) x}\left(c_{2} c_{3} e^{(\beta+\gamma) x}+c_{3} c_{4} e^{(\gamma+\delta) x}+c_{4} c_{2} e^{(\delta+\beta) x}\right) .
\end{aligned}
$$

Theorem 5.3. For $n \geq 0$,

$$
\begin{aligned}
B t_{n}^{(2)}= & \sum_{\substack{k_{1}+\cdots+k_{5}=n \\
k_{1}, \ldots, k_{5} \geq 0}}\left(\begin{array}{c}
n \\
k_{1}, \ldots, k_{5}
\end{array}\right) \mathfrak{T}_{k_{1}} \cdots \mathfrak{T}_{k_{5}}-\frac{A}{563^{2}} 5^{n} \mathfrak{T}_{n}^{(500,1423,2598,4986)}-\cdots \\
& -S \sum_{\substack{k_{1}+k_{2}+k_{3}+k_{4}=n \\
k_{1}, k_{2}, k_{3}, k_{4} \geq 0}}\left(\begin{array}{c}
n \\
k_{1}, k_{2}, k_{3}, k_{4}
\end{array}\right) t_{k_{1}} \mathfrak{T}_{k_{2}} \mathfrak{T}_{k_{3}} \mathfrak{T}_{k_{4}},
\end{aligned}
$$

where

$$
\begin{aligned}
& A=-G-J-M+2 N-P-Q-3 S+6, \\
& B=-2 G-K-2 M-2 N-5 P-2 Q-6 R-12 S+30, \\
& E=G+J+M-N+2 P+2 Q+3 R+6 S-15, \\
& F=-3 G-J-3 K-7 M-12 P-3 Q-6 R-27 S+60, \\
& H=-L-2 N-P-Q-4 R-3 S+10, \\
& I=-G-J-2 L-M-2 P-3 Q-6 R-7 S+20,
\end{aligned}
$$

$t_{n}$ and $t_{n}^{(1)}$ are same as those in Lemma 3.2 and Theorem 4.8, respectively. 
Remark. If $G=J=K=L=M=N=P=Q=R=S=0$, then by $A=6, B=30$, $E=-15, F=60, H=10$ and $I=20$, we have for $n \geq 0$,

$$
\begin{aligned}
30 t_{n}^{(2)}= & \sum_{\substack{k_{1}+\ldots+k_{5}=n \\
k_{1}, \ldots, k_{5} \geq 0}}\left(\begin{array}{c}
n \\
k_{1}, \ldots, k_{5}
\end{array}\right) \mathfrak{T}_{k_{1}} \ldots \mathfrak{T}_{k_{5}}-\frac{6}{563^{2}} 5^{n} \mathfrak{T}_{n}^{(500,1423,2598,4986)} \\
& +\frac{15}{563^{2}} \sum_{k=0}^{n}\left(\begin{array}{l}
n \\
k
\end{array}\right) 4^{n-k} \mathfrak{T}_{n-k}^{(3052,4658,8804,16451)} \mathfrak{T}_{k}+\frac{60}{563} \sum_{k=0}^{n}\left(\begin{array}{l}
n \\
k
\end{array}\right) \mathfrak{T}_{k} \\
& -\frac{10}{563^{2}} \sum_{k=0}^{n}\left(\begin{array}{l}
n \\
k
\end{array}\right) 3^{n-k} 2^{k} \mathfrak{T}_{n-k}^{(15,27,48,107)} \mathfrak{T}_{k}^{(40,64,215,344)}-\frac{20}{563} \sum_{k=0}^{n}\left(\begin{array}{l}
n \\
k
\end{array}\right) 3^{k} \mathfrak{T}_{k}^{(15,27,48,107)} t_{n-k} .
\end{aligned}
$$

\subsection{Case 3}

Let $C \neq 0, B=D=0$, we can obtain the following theorem.

Let

$$
\begin{aligned}
& \sum_{n=0}^{\infty} t_{n}^{(3)} \frac{x^{n}}{n !} \\
& =c_{1} c_{2} c_{3} e^{(\alpha+\beta+\gamma) x}\left(c_{1}^{2} e^{2 \alpha x}+c_{2}^{2} e^{2 \beta x}+c_{3}^{2} e^{2 \gamma x}\right)+\cdots \\
& \quad+c_{2} c_{3} c_{4} e^{(\beta+\gamma+\delta) x}\left(c_{2}^{2} e^{2 \beta x}+c_{3}^{2} e^{2 \gamma x}+c_{4}^{2} e^{2 \delta x}\right) .
\end{aligned}
$$

Theorem 5.4. For $n \geq 0$,

$$
\begin{aligned}
C t_{n}^{(3)}= & \sum_{\substack{k_{1}+\cdots+k_{5}=n \\
k_{1}, \ldots, k_{5} \geq 0}}\left(\begin{array}{c}
n \\
k_{1}, \ldots, k_{5}
\end{array}\right) \mathfrak{T}_{k_{1}} \cdots \mathfrak{T}_{k_{5}}-\frac{A}{563^{2}} 5^{n} \mathfrak{T}_{n}^{(500,1423,2598,4986)}-\cdots \\
& -S \sum_{\substack{k_{1}+k_{2}+k_{3}+k_{4}=n \\
k_{1}, k_{2}, k_{3}, k_{4} \geq 0}}\left(\begin{array}{c}
n \\
k_{1}, k_{2}, k_{3}, k_{4}
\end{array}\right) t_{k_{1}} \mathfrak{T}_{k_{2}} \mathfrak{T}_{k_{3}} \mathfrak{T}_{k_{4}},
\end{aligned}
$$

where

$$
\begin{aligned}
& A=I+2 L+2 N+P+2 Q+6 R+4 S-14, \\
& C=-G-I-J-2 L-M-2 P-3 Q-6 R-7 S+20, \\
& E=-I-2 L-N-Q-3 R-S+5, \\
& F=3 G-J-M+6 N+3 P+3 Q+12 R+9 S-30, \\
& H=-L-2 N-P-Q-4 R-3 S+10, \\
& K=-2 G-2 M-2 N-5 P-2 Q-6 R-12 S+30,
\end{aligned}
$$

$t_{n}$ and $t_{n}^{(1)}$ are same as those in Lemma 3.2 and Theorem 4.8, respectively.

Remark. If $G=I=J=L=M=N=P=Q=R=S=0$, then by $A=-14, C=20$, $E=5, F=-30, H=10$ and $K=30$, we have for $n \geq 0$,

$$
20 t_{n}^{(3)}=\sum_{\substack{k_{1}+\ldots+k_{5}=n \\
k_{1}, \ldots, k_{5} \geq 0}}\left(\begin{array}{c}
n \\
k_{1}, \ldots, k_{5}
\end{array}\right) \mathfrak{T}_{k_{1}} \cdots \mathfrak{T}_{k_{5}}+\frac{14}{563^{2}} 5^{n} \mathfrak{T}_{n}^{(500,1423,2598,4986)}
$$




$$
\begin{aligned}
& -\frac{5}{563^{2}} \sum_{k=0}^{n}\left(\begin{array}{l}
n \\
k
\end{array}\right) 4^{n-k} \mathfrak{T}_{n-k}^{(3052,4658,8804,16451)} \mathfrak{T}_{k}+\frac{30}{563} \sum_{k=0}^{n}\left(\begin{array}{l}
n \\
k
\end{array}\right) \mathfrak{T}_{k} \\
& -\frac{10}{563^{2}} \sum_{k=0}^{n}\left(\begin{array}{l}
n \\
k
\end{array}\right) 3^{n-k} 2^{k} \mathfrak{T}_{n-k}^{(15,27,48,107)} \mathfrak{T}_{k}^{(40,64,215,344)} \\
& +\frac{30}{563} \sum_{\substack{k_{1}+k_{2}+k_{3}=n \\
k_{1}, k_{2}, k_{3} \geq 0}}\left(\begin{array}{c}
n \\
k_{1}, k_{2}, k_{3}
\end{array}\right)(-1)^{k_{1}} \mathfrak{T}_{k_{1}}^{(-5,2,13,32)} t_{k_{2}} .
\end{aligned}
$$

\subsection{Case 4}

Let $D \neq 0, B=C=0$, we can obtain the following theorem.

Let

$$
\begin{aligned}
& \sum_{n=0}^{\infty} t_{n}^{(4)} \frac{x^{n}}{n !} \\
& =c_{1} c_{2} c_{3} e^{(\alpha+\beta+\gamma) x}\left(c_{1} e^{\alpha x}+c_{2} e^{\beta x}+c_{3} e^{\gamma x}\right)^{2}+\cdots \\
& \quad+c_{2} c_{3} c_{4} e^{(\beta+\gamma+\delta) x}\left(c_{2} e^{\beta x}+c_{3} e^{\gamma x}+c_{4} e^{\delta x}\right)^{2} .
\end{aligned}
$$

Theorem 5.5. For $n \geq 0$,

$$
\begin{aligned}
D t_{n}^{(4)}= & \sum_{\substack{k_{1}+\cdots+k_{5}=n \\
k_{1}, \ldots, k_{5} \geq 0}}\left(\begin{array}{c}
n \\
k_{1}, \ldots, k_{5}
\end{array}\right) \mathfrak{T}_{k_{1}} \cdots \mathfrak{T}_{k_{5}}-\frac{A}{563^{2}} 5^{n} \mathfrak{T}_{n}^{(500,1423,2598,4986)}-\cdots \\
& -S \sum_{\substack{k_{1}+k_{2}+k_{3}+k_{4}=n \\
k_{1}, k_{2}, k_{3}, k_{4} \geq 0}}\left(\begin{array}{c}
n \\
k_{1}, k_{2}, k_{3}, k_{4}
\end{array}\right) t_{k_{1}} \mathfrak{T}_{k_{2}} \mathfrak{T}_{k_{3}} \mathfrak{T}_{k_{4}},
\end{aligned}
$$

where

$$
\begin{aligned}
& A=I+2 L+2 N+P+2 Q+6 R+4 S-14, \\
& D=-G-I-J-2 L-M-2 P-3 Q-6 R-7 S+20, \\
& E=-I-2 L-N-Q-3 R-S+5, \\
& F=-3 G-6 I-7 J-7 M+6 N-12 L-9 P-15 Q-24 R-33 S+90, \\
& H=-L-2 N-P-Q-4 R-3 S+10, \\
& K=2 I+2 J-2 N+4 L-P+4 Q+6 R+2 S-10,
\end{aligned}
$$

$t_{n}$ and $t_{n}^{(1)}$ are given in Lemma 3.2 and Theorem 4.8, respectively.

Remark. If $G=I=J=L=M=N=P=Q=R=S=0$, then by $A=-14, D=20$, $E=5, F=90, H=10$ and $K=-10$, we have for $n \geq 0$,

$$
\begin{aligned}
20 t_{n}^{4}= & \sum_{\substack{k_{1}+\cdots+k_{5}=n \\
k_{1}, \ldots, k_{5} \geq 0}}\left(\begin{array}{c}
n \\
k_{1}, \ldots, k_{5}
\end{array}\right) \mathfrak{T}_{k_{1}} \cdots \mathfrak{T}_{k_{5}}+\frac{14}{563^{2}} 5^{n} \mathfrak{T}_{n}^{(500,1423,2598,4986)} \\
& -\frac{5}{563^{2}} \sum_{k=0}^{n}\left(\begin{array}{l}
n \\
k
\end{array}\right) 4^{n-k} \mathfrak{T}_{n-k}^{(3052,4658,8804,16451)} \mathfrak{T}_{k}+\frac{90}{563} \sum_{k=0}^{n}\left(\begin{array}{l}
n \\
k
\end{array}\right) \mathfrak{T}_{k}
\end{aligned}
$$




$$
\begin{aligned}
& -\frac{10}{563^{2}} \sum_{k=0}^{n}\left(\begin{array}{l}
n \\
k
\end{array}\right) 3^{n-k} 2^{k} \mathfrak{T}_{n-k}^{(15,27,48,107)} \mathfrak{T}_{k}^{(40,64,215,344)} \\
& -\frac{10}{563} \sum_{\substack{k_{1}+k_{2}+k_{3}=n \\
k_{1}, k_{2}, k_{3} \geq 0}}\left(\begin{array}{c}
n \\
k_{1}, k_{2}, k_{3}
\end{array}\right)(-1)^{k_{1}} \mathfrak{T}_{k_{1}}^{(-5,2,13,32)} t_{k_{2}} .
\end{aligned}
$$

\section{More general results}

We shall consider a general case of Lemmata 3.1, 4.4 and 4.6. Similarly to the proof of Lemma 3.1, for Tetranacci-type numbers $s_{1, n}^{(n)}$, satisfying the recurrence relation $s_{1, k}^{(n)}=s_{1, k-1}^{(n)}+s_{1, k-2}^{(n)}+s_{1, k-3}^{(n)}+$ $s_{1, k-4}^{(n)}(k \geq 4)$ with given initial values $s_{1,0}^{(n)}, s_{1,1}^{(n)}, s_{1,2}^{(n)}$ and $s_{1,3}^{(n)}$, we have the form

$$
d_{1}^{(n)} e^{\alpha x}+d_{2}^{(n)} e^{\beta x}+d_{3}^{(n)} e^{\gamma x}+d_{4}^{(n)} e^{\delta x}=\sum_{k=0}^{\infty} s_{1, k}^{(n)} \frac{x^{k}}{k !} .
$$

Theorem 6.1. For $n \geq 1$, we have

$$
c_{1}^{n} e^{\alpha x}+c_{2}^{n} e^{\beta x}+c_{3}^{n} e^{\gamma x}+c_{4}^{n} e^{\delta x}=\frac{1}{A_{1}^{(n)}} \sum_{k=0}^{\infty} \mathfrak{T}_{1, k}^{\left(s_{1,0}^{(n)}, s_{1,1}^{(n)}, s_{1,2}^{(n)}, s_{1,3}^{(n)}\right)} \frac{x^{k}}{k !},
$$

where $s_{1,0}^{(n)}, s_{1,1}^{(n)}, s_{1,2}^{(n)}, s_{1,3}^{(n)}$ and $A_{1}^{(n)}$ satisfy the recurrence relation:

$$
\begin{aligned}
& s_{1,0}^{(n)}= \pm \operatorname{lcm}\left(b_{1}, b_{2}, b_{3}\right), \quad s_{1,1}^{(n)}=M s_{1,0}^{(n)}, \quad s_{1,2}^{(n)}=N s_{1,0}^{(n)}, \quad s_{1,3}^{(n)}=P s_{1,0}^{(n)}, \\
& A_{1}^{(n)}=\frac{A_{1}^{(n-1)}}{s_{1,2}^{(n-1)}}\left(4 s_{1,3}^{(n)}-3 s_{1,2}^{(n)}-2 s_{1,1}^{(n)}-s_{1,0}^{(n)}\right)
\end{aligned}
$$

$b_{1}, b_{2}, b_{3}, M, N$ and $P$ are determined in the proof.

Proof. By $d_{1}^{(n)}=A_{1}^{(n)} c_{1}^{n}, d_{1}^{(n-1)}=A_{1}^{(n-1)} c_{1}^{n-1}$,

$$
\begin{aligned}
d_{1}^{(n)} & =\frac{s_{1,0}^{(n)} \beta \gamma \delta+s_{1,2}^{(n)}(\beta+\gamma+\delta)-s_{1,3}^{(n)}-s_{1,1}^{(n)}(\beta \gamma+\beta \delta+\gamma \delta)}{(\beta-\alpha)(\gamma-\alpha)(\delta-\alpha)} \\
c_{1}= & \frac{2-(\beta+\gamma+\delta)+(\beta \gamma+\gamma \delta+\delta \beta)}{(\alpha-\beta)(\alpha-\gamma)(\alpha-\delta)} \\
= & \frac{\alpha^{2}}{(\alpha-\beta)(\alpha-\gamma)(\alpha-\delta)} \\
= & \frac{1}{-\alpha^{3}+6 \alpha-1}
\end{aligned}
$$

we can obtain the following recurrence relation:

$$
\begin{aligned}
& A=-3 s_{1,1}^{(n-1)}-s_{1,2}^{(n-1)}+s_{1,3}^{(n-1)}, \quad B=5 s_{1,1}^{(n-1)}+5 s_{1,2}^{(n-1)}-5 s_{1,3}^{(n-1)}, \\
& C=5 s_{1,1}^{(n-1)}-2 s_{1,2}^{(n-1)}+2 s_{1,3}^{(n-1)}, \quad D=5 s_{1,1}^{(n-1)}-s_{1,2}^{(n-1)}+s_{1,3}^{(n-1)}, \\
& E=s_{1,0}^{(n-1)}-s_{1,1}^{(n-1)}, \quad F=-5 s_{1,0}^{(n-1)}, \quad G=2 s_{1,0}^{(n-1)}+6 s_{1,1}^{(n-1)}, \\
& H=s_{1,0}^{(n-1)}-s_{1,1}^{(n-1)}, \quad I=4 s_{1,1}^{(n-1)}+6 s_{1,2}^{(n-1)}, \quad J=-3 s_{1,1}^{(n-1)}-5 s_{1,2}^{(n-1)},
\end{aligned}
$$




$$
\begin{aligned}
& K=-2 s_{1,1}^{(n-1)}+2 s_{1,2}^{(n-1)}, \quad L=-s_{1,1}^{(n-1)}+s_{1,2}^{(n-1)}, \\
& M=\frac{(L A-D I)(F A-B E)-(H A-D E)(J A-B I)}{(G A-C E)(J A-B I)-(K A-C I)(F A-B E)}, \\
& N=\frac{M(K A-C I)+(L A-D I)}{B I-J A}, \quad P=-\frac{1}{A}(B N+C M+D), \\
& M=\frac{a_{1}}{b_{1}}, \quad N=\frac{a_{2}}{b_{2}}, \quad P=\frac{a_{3}}{b_{3}}, \quad \text { with } \quad \operatorname{gcd}\left(a_{i}, b_{i}\right)=1, \\
& s_{1,0}^{(n)}= \pm \operatorname{lcm}\left(b_{1}, b_{2}, b_{3}\right), \quad s_{1,1}^{(n)}=M s_{1,0}^{(n)}, \quad s_{1,2}^{(n)}=N s_{1,0}^{(n)}, \quad s_{1,3}^{(n)}=P s_{1,0}^{(n)}, \\
& A_{1}^{(n)}=\frac{A_{1}^{(n-1)}}{s_{1,2}^{(n-1)}}\left(4 s_{1,3}^{(n)}-3 s_{1,2}^{(n)}-2 s_{1,1}^{(n)}-s_{1,0}^{(n)}\right) .
\end{aligned}
$$

We choose the value of $s_{1,0}^{(n)}$ such that for some $k_{0}, \forall k \geq k_{0}, \mathfrak{T}_{1, k}^{\left(s_{1,0}^{(n)}, s_{1,1}^{(n)}, s_{1,2}^{(n)}, s_{1,3}^{(n)}\right)}$ is positive.

Next we shall consider a general case of Lemma 3.3. Similarly to the proof of Lemma 3.3, for Tetranacci-type numbers $s_{1, k}^{(n)}$, satisfying the recurrence relation $s_{1, k}^{(n)}=s_{1, k-1}^{(n)}+s_{1, k-2}^{(n)}+s_{1, k-3}^{(n)}+$ $s_{1, k-4}^{(n)}(k \geq 4)$ with given initial values $s_{1,0}^{(n)}, s_{1,1}^{(n)}, s_{1,2}^{(n)}$ and $s_{1,3}^{(n)}$, we have the form

$$
r_{1}^{(n)} e^{\alpha x}+r_{2}^{(n)} e^{\beta x}+r_{3}^{(n)} e^{\gamma x}+r_{4}^{(n)} e^{\delta x}=\sum_{k=0}^{\infty} s_{1, k}^{(n)} \frac{x^{k}}{k !},
$$

where $r_{1}^{(n)}, r_{2}^{(n)}, r_{3}^{(n)}$ and $r_{4}^{(n)}$ are determined by solving the system of the equations.

\section{Theorem 6.2.}

$$
c_{2}^{n} c_{3}^{n} c_{4}^{n} e^{\alpha x}+c_{1}^{n} c_{3}^{n} c_{4}^{n} e^{\beta x}+c_{1}^{n} c_{2}^{n} c_{4}^{n} e^{\gamma x}+c_{1}^{n} c_{2}^{n} c_{3}^{n} e^{\delta x}=\frac{1}{A_{2}^{(n)}} \sum_{k=0}^{\infty} \mathfrak{T}_{2, k}^{\left(s_{2,0}^{(n)}, s_{2,1}^{(n)}, s_{2,2}^{(n)}, s_{2,3}^{(n)}\right)} \frac{x^{k}}{k !},
$$

where $s_{2,0}^{(n)}, s_{2,1}^{(n)}, s_{2,2}^{(n)}, s_{2,3}^{(n)}$ and $A_{2}^{(n)}$ satisfy the recurrence relation:

$$
\begin{aligned}
& s_{2,0}^{(n)}= \pm \operatorname{lcm}\left(b_{1}, b_{2}, b_{3}\right), \quad s_{2,1}^{(n)}=M s_{2,0}^{(n)}, \quad s_{2,2}^{(n)}=N s_{2,0}^{(n)}, \quad s_{2,3}^{(n)}=P s_{2,0}^{(n)}, \\
& A_{2}^{(n)}=\frac{A_{2}^{(n-1)}}{2 s_{2,2}^{(n-1)}-s_{2,3}^{(n-1)}}\left(-16 s_{2,3}^{(n)}+103 s_{2,2}^{(n)}-157 s_{2,1}^{(n)}-10 s_{2,0}^{(n)}\right)
\end{aligned}
$$

$b_{1}, b_{2}, b_{3}, M, N$ and $P$ are determined in the proof.

Proof. By $r_{1}^{(n)}=A_{2}^{(n)} c_{2}^{n} c_{3}^{n} c_{4}^{n}$, we can obtain the following recurrence relation:

$$
\begin{aligned}
& A=-16 s_{2,0}^{(n-1)}-16 s_{2,1}^{(n-1)}+158 s_{2,2}^{(n-1)}-71 s_{2,3}^{(n-1)}, \\
& B=103 s_{2,0}^{(n-1)}+103 s_{2,1}^{(n-1)}-243 s_{2,2}^{(n-1)}+70 s_{2,3}^{(n-1)}, \\
& C=-157 s_{2,0}^{(n-1)}-157 s_{2,1}^{(n-1)}-209 s_{2,2}^{(n-1)}+183 s_{2,3}^{(n-1)}, \\
& D=-10 s_{2,0}^{(n-1)}-10 s_{2,1}^{(n-1)}-42 s_{2,2}^{(n-1)}+26 s_{2,3}^{(n-1)}, \\
& E=32 s_{2,0}^{(n-1)}+16 s_{2,1}^{(n-1)}-330 s_{2,2}^{(n-1)}+157 s_{2,3}^{(n-1)}, \\
& F=-206 s_{2,0}^{(n-1)}-103 s_{2,1}^{(n-1)}+365 s_{2,2}^{(n-1)}-131 s_{2,3}^{(n-1)},
\end{aligned}
$$




$$
\begin{aligned}
& G=314 s_{2,0}^{(n-1)}+157 s_{2,1}^{(n-1)}+351 s_{2,2}^{(n-1)}-254 s_{2,3}^{(n-1)}, \\
& H=20 s_{2,0}^{(n-1)}+10 s_{2,1}^{(n-1)}+216 s_{2,2}^{(n-1)}-113 s_{2,3}^{(n-1)}, \\
& I=32 s_{2,1}^{(n-1)}-36 s_{2,2}^{(n-1)}+10 s_{2,3}^{(n-1)}, \quad J=-206 s_{2,1}^{(n-1)}+91 s_{2,2}^{(n-1)}+6 s_{2,3}^{(n-1)}, \\
& K=314 s_{2,1}^{(n-1)}+69 s_{2,2}^{(n-1)}-113 s_{2,3}^{(n-1)}, \quad L=20 s_{2,1}^{(n-1)}-304 s_{2,2}^{(n-1)}+147 s_{2,3}^{(n-1)}, \\
& M=\frac{(L A-D I)(F A-B E)-(H A-D E)(J A-B I)}{(G A-C E)(J A-B I)-(K A-C I)(F A-B E)}, \\
& N=\frac{M(G A-C E)+(H A-D E)}{B E-F A}, \quad P=-\frac{1}{A}(B N+C M+D), \\
& M=\frac{a_{1}}{b_{1}} \quad N=\frac{a_{2}}{b_{2}}, \quad P=\frac{a_{3}}{b_{3}}, \quad \operatorname{gcd}\left(a_{i}, b_{i}\right)=1, \\
& s_{2,0}^{(n)}= \pm \frac{1 c m\left(b_{1}, b_{2}, b_{3}\right), \quad s_{2,1}^{(n)}=M s_{2,0}^{(n)}, \quad s_{2,2}^{(n)}=N s_{2,0}^{(n)}, \quad s_{2,3}^{(n)}=P s_{2,0}^{(n)},}{A_{2}^{(n)}=\frac{A_{2}^{(n-1)}}{2 s_{2,2}^{(n-1)}-s_{2,3}^{(n-1)}}\left(-16 s_{2,3}^{(n)}+103 s_{2,2}^{(n)}-157 s_{2,1}^{(n)}-10 s_{2,0}^{(n)}\right) .}
\end{aligned}
$$

We choose the value of $s_{2,0}^{(n)}$ such that for some $k_{0}, \forall k \geq k_{0}, \mathfrak{T}_{2, k}^{\left(s_{2,0}^{(n)}, s_{2,1}^{(n)}, s_{2,2}^{(n)}, s_{2,3}^{(n)}\right)}$ is positive.

As application, we compute some values of $s_{2,0}^{(n)}, s_{2,1}^{(n)}, s_{2,2}^{(n)}, A_{2}^{(n)}$ for some $n$. For $n=2$, we have

$$
\begin{aligned}
& A=-170, \quad B=-1228, \quad C=3610, \quad D=316, \quad E=606, \quad F=1377, \\
& G=-4821, \quad H=-888, \quad I=-84, \quad J=963, \quad K=-2091, \quad L=792, \\
& M=-\frac{34}{15}, \quad N=-6, \quad P=-\frac{44}{15}, \\
& s_{2,0}^{2}=-15, \quad s_{2,1}^{2}=34, \quad s_{2,2}^{2}=90, \quad s_{2,3}^{2}=44, \quad A_{2}^{2}=563^{2}, \\
& c_{2}^{2} c_{3}^{2} c_{4}^{2} e^{\alpha x}+c_{1}^{2} c_{3}^{2} c_{4}^{2} e^{\beta x}+c_{1}^{2} c_{2}^{2} c_{4}^{2} e^{\gamma x}+c_{1}^{2} c_{2}^{2} c_{3}^{2} e^{\delta x}=\frac{1}{563^{2}} \sum_{k=0}^{\infty} \mathfrak{T}_{2, k}^{(-15,34,90,44)} \frac{x^{k}}{k !} .
\end{aligned}
$$

For $n=3$, we have

$A=10792, \quad B=-16833, \quad C=13741, \quad D=-2826, \quad E=-22728, \quad F=26674$,

$G=21042, \quad H=14508, \quad I=-1712, \quad J=1450, \quad K=11914, \quad L=-20212$,

$M=\frac{353}{175}, \quad N=-\frac{21}{25}, \quad P=\frac{38}{25}$,

$s_{2,0}^{3}=175, \quad s_{2,1}^{3}=353, \quad s_{2,2}^{3}=-147, \quad s_{2,3}^{3}=266, \quad A_{2}^{3}=-563^{3}$,

$c_{2}^{3} c_{3}^{3} c_{4}^{3} e^{\alpha x}+c_{1}^{3} c_{3}^{3} c_{4}^{3} e^{\beta x}+c_{1}^{3} c_{2}^{3} c_{4}^{3} e^{\gamma x}+c_{1}^{3} c_{2}^{3} c_{3}^{3} e^{\delta x}=-\frac{1}{563^{3}} \sum_{k=0}^{\infty} \mathfrak{T}_{2, k}^{(175,353,-147,266)} \frac{x^{k}}{k !}$.

We can obtain more convolution identities for any fixed $n$, but we only give some of the results. The proofs of the next eight theorems are similar to the proofs of Theorem (Lemma) 3.2, 4.5, 4.7, 4.8, 5.2, 5.3, 5.4 and 5.5, and, hence, omitted. 
Let

$$
c_{1}^{n} c_{2}^{n} e^{(\alpha+\beta) x}+\cdots+c_{3}^{n} c_{4}^{n} e^{(\gamma+\delta) x}=\sum_{k=0}^{\infty} t_{1, k}^{(n)} \frac{x^{k}}{k !},
$$

then by previous algebraic identities, we can obtain the following theorems.

Theorem 6.3. For $m \geq 0, n \geq 1$,

$$
c_{1}^{n} c_{2}^{n} e^{(\alpha+\beta) x}+\cdots+c_{3}^{n} c_{4}^{n} e^{(\gamma+\delta) x}=\sum_{k=0}^{\infty} t_{1, m}^{(n)} \frac{x^{m}}{m !},
$$

where

$$
t_{1, m}^{(n)}=\frac{1}{2}\left(\frac{1}{\left(A_{1}^{(n)}\right)^{2}} \sum_{k=0}^{m}\left(\begin{array}{c}
m \\
k
\end{array}\right) \mathfrak{T}_{1, k}^{\left(s_{1,0}^{n}, s_{1,1}^{(n)}, s_{1,2}^{(n)}, s_{1,3}^{(n)}\right)} \mathfrak{T}_{1, m-k}^{\left(s_{1,0}^{(n)}, s_{1,1}^{(n)}, s_{1,2}^{(n)}, s_{1,3}^{(n)}\right)}-\frac{2^{m}}{A_{1}^{(2 n)}} \mathfrak{T}_{1, m}^{\left(s_{1,0}^{(n)}, s_{1,1}^{(n)}, s_{1,2}^{(n)}, s_{1,3}^{(n)}\right)}\right) .
$$

Theorem 6.4. For $m \geq 0, n \geq 1$,

$$
\begin{aligned}
& \frac{1}{\left(A_{1}^{(n)}\right)^{3}} \sum_{\substack{k_{1}+k_{2}+k_{3}=m \\
k_{1}, k_{2}, k_{3} \geq 0}}\left(\begin{array}{c}
m \\
k_{1}, k_{2}, k_{3}
\end{array}\right) \mathfrak{T}_{1, k_{1}}^{\left(s_{1,0}^{(n)}, s_{1,1}^{(n)}, s_{1,2}^{(n)}, s_{1,3}^{(n)}\right)} \mathfrak{T}_{1, k_{2}}^{\left(s_{1,0}^{(n)}, s_{1,1}^{(n)}, s_{1,2}^{(n)}, s_{1,3}^{(n)}\right)} \mathfrak{T}_{1, k_{3}}^{\left(s_{1,0}^{(n)}, s_{1,1}^{(n)}, s_{1,2}^{(n)}, s_{1,3}^{(n)}\right)} \\
& =\frac{A}{A_{1}^{(3 n)}} 3^{m} \mathfrak{T}_{1, m}^{\left(s_{1,0}^{(3 n)}, s_{1,1}^{(3 n)}, s_{1,2}^{(3 n)}, s_{1,3}^{(3 n)}\right)}+\frac{B}{A_{2}^{(n)}} \sum_{k=0}^{m}\left(\begin{array}{c}
m \\
k
\end{array}\right) \mathfrak{T}_{2, k}^{\left(s_{2,0}^{(n)}, s_{2,1}^{(n)}, s_{2,2}^{(n)}, s_{2,3}^{(n)}\right)}(-1)^{k} \\
& +\frac{C}{A_{1}^{(n)} A_{1}^{(2 n)}} \sum_{k=0}^{m}\left(\begin{array}{c}
m \\
k
\end{array}\right) \mathfrak{T}_{1, k}^{\left(s_{1,0}^{(n)}, s_{1,1}^{(n)}, s_{1,2}^{(n)}, s_{1,3}^{(n)}\right)} 2^{m-k} \mathfrak{T}_{1, m-k}^{\left(s_{1,0}^{(2 n)}, s_{1,1}^{(2 n)}, s_{1,2}^{(2 n)}, s_{1,3}^{(2 n)}\right)} \\
& +\frac{D}{A_{1}^{(n)}} \sum_{k=0}^{m}\left(\begin{array}{c}
m \\
k
\end{array}\right) \mathfrak{T}_{1, k}^{\left(s_{1,0}^{(n)}, s_{1,1}^{(n)}, s_{1,2}^{(n)}, s_{1,3}^{(n)}\right)} t_{1, m-k}^{(n)},
\end{aligned}
$$

where

$$
A=D-2, \quad B=-3 D+6, \quad C=-D+3,
$$

$t_{1, m}^{(n)}$ is determined in Theorem 6.3.

Theorem 6.5. For $m \geq 0, n \geq 1$,

$$
\begin{aligned}
& \frac{1}{\left(A_{1}^{(n)}\right)^{4}} \sum_{\substack{k_{1}+k_{2}+k_{3}+k_{4}=m \\
k_{1}, k_{2}, k_{3}, k_{4} \geq 0}}\left(\begin{array}{c}
m \\
k_{1}, k_{2}, k_{3}, k_{4}
\end{array}\right) \mathfrak{T}_{1, k_{1}}^{\left(s_{1,0}^{(n)}, s_{1,1}^{(n)}, s_{1,2}^{(n)}, s_{1,3}^{(n)}\right)} \cdots \mathfrak{T}_{1, k_{4}}^{\left(s_{1,0}^{(n)}, s_{1,1}^{(n)}, s_{1,2}^{(n)}, s_{1,3}^{(n)}\right)} \\
& =\frac{A}{A_{1}^{(4 n)}} 4^{m} \mathfrak{T}_{1, m}^{\left(s_{1,0}^{(4 n)}, \cdots, s_{1,3}^{(4 n)}\right)}+B\left(\frac{-1}{563}\right)^{n}+\frac{C}{A_{1}^{(3 n)} A_{1}^{(n)}} \sum_{k=0}^{m}\left(\begin{array}{c}
m \\
k
\end{array}\right) 3^{m-k} \mathfrak{T}_{1, m-k}^{\left(s_{1,0}^{(3 n)}, \cdots, s_{1,3}^{(3 n)}\right)} \mathfrak{T}_{1, k}^{\left(s_{1,0}^{(n)}, \cdots, s_{1,3}^{(n)}\right)} \\
& \quad+\frac{D}{\left(A_{1}^{(2 n)}\right)^{2}} \sum_{k=0}^{m}\left(\begin{array}{c}
m \\
k
\end{array}\right) 2^{m} \mathfrak{T}_{1, k}^{\left(s_{1,0}^{(2 n)}, \cdots, s_{1,3}^{(2 n)}\right)} \mathfrak{T}_{1, m-k}^{\left(s_{1,0}^{(2 n)}, \cdots, s_{1,3}^{(2 n)}\right)} \\
& \quad+\frac{E}{A_{1}^{(2 n)} \sum_{k=0}^{m}\left(\begin{array}{c}
m \\
k
\end{array}\right) 2^{m-k} \mathfrak{T}_{1, m-k}^{\left(s_{1,0}^{(2 n)}, \cdots, s_{1,3}^{(2 n)}\right)} t_{1, m-k}^{(n)}+F \sum_{k=0}^{m}\left(\begin{array}{c}
m \\
k
\end{array}\right) t_{1, k}^{(n)} t_{1, m-k}^{(n)}} \\
& \quad+\frac{G}{A_{1}^{(2 n)}\left(A_{1}^{(n)}\right)^{2}} \sum_{\substack{k_{1}+k_{2}+k_{3}=m \\
k_{1}, k_{2}, k_{3} \geq 0}}\left(\begin{array}{c}
m \\
k_{1}, k_{2}, k_{3}
\end{array}\right) \mathfrak{T}_{1, k_{1}}^{\left(s_{1,0}^{(2 n)}, \cdots, s_{1,3}^{(2 n)}\right)} 2^{k_{1}} \mathfrak{T}_{1, k_{2}}^{\left(s_{1,0}^{(n)}, \cdots, s_{1,3}^{(n)}\right)} \mathfrak{T}_{1, k_{3}}^{\left(s_{1,0}^{(n)}, \cdots, s_{1,3}^{(n)}\right)}
\end{aligned}
$$




$$
\begin{aligned}
& +\frac{H}{\left(A_{1}^{(n)}\right)^{2}} \sum_{\substack{k_{1}+k_{2}+k_{3}=m \\
k_{1}, k_{2}, k_{3} \geq 0}}\left(\begin{array}{c}
m \\
k_{1}, k_{2}, k_{3}
\end{array}\right) t_{1, k_{1}}^{(n)} \mathfrak{T}_{1, k_{2}}^{\left(s_{1,0}^{(n)}, \cdots, s_{1,3}^{(n)}\right)} \mathfrak{T}_{1, k_{3}}^{\left(s_{1,0}^{(n)}, \cdots, s_{1,3}^{(n)}\right)} \\
& +\frac{J}{A_{2}^{(n)} A_{1}^{(n)}} \sum_{\substack{k_{1}+k_{2}+k_{3}=m \\
k_{1}, k_{2}, k_{3} \geq 0}}\left(\begin{array}{c}
m \\
k_{1}, k_{2}, k_{3}
\end{array}\right)(-1)^{k_{1}} \mathfrak{T}_{2, k_{1}}^{\left(s_{2,0}^{(n)}, \cdots, s_{2,3}^{(n)}\right)} \mathfrak{T}_{1, k_{2}}^{\left(s_{1,0}^{(n)}, \cdots, s_{1,3}^{(n)}\right)},
\end{aligned}
$$

where $A=-D+E+G+H-3, \quad B=-4 D+4 E+4 G+4 H-12, \quad C=-E-2 G-H+4$, $F=-2 D-2 G-2 H+6, \quad J=4 D-E+2 G-H, t_{1, m}^{(n)}$ is determined in Theorem 6.3.

Let

$$
\begin{aligned}
& \sum_{k=0}^{\infty} t_{2, k}^{(n)} \frac{x^{k}}{k !}=c_{1}^{n} c_{2}^{n} c_{3}^{n} e^{(\alpha+\beta+\gamma) x}\left(c_{1}^{n} e^{\alpha x}+c_{2}^{n} e^{\beta x}+c_{3}^{n} e^{\gamma x}\right)+\cdots \\
& \quad+c_{2}^{n} c_{3}^{n} c_{4}^{n} e^{(\alpha+\gamma+\delta) x}\left(c_{2}^{n} e^{\alpha x}+c_{3}^{n} e^{\gamma x}+c_{4}^{n} e^{\delta x}\right) .
\end{aligned}
$$

Theorem 6.6. For $m \geq 0, n \geq 1, I \neq 0$,

$$
\begin{aligned}
& I t_{2, m}^{(n)}=\frac{1}{\left(A_{1}^{(n)}\right)^{4}} \sum_{\substack{k_{1}+k_{2}+k_{3}+k_{4}=m \\
k_{1}, k_{2}, k_{3}, k_{4} \geq 0}}\left(\begin{array}{c}
m \\
k_{1}, k_{2}, k_{3}, k_{4}
\end{array}\right) \mathfrak{T}_{1, k_{1}}^{\left(s_{1,0}^{(n)}, \cdots, s_{1,3}^{(n)}\right)} \cdots \mathfrak{T}_{1, k_{4}}^{\left(s_{1,0}^{(n)}, \cdots, s_{1,3}^{(n)}\right)} \\
& -\frac{A}{A_{1}^{(4 n)} 4^{m} \mathfrak{T}_{1, m}^{\left(s_{1,0}^{(4 n)}, \cdots, s_{1,3}^{(n)}\right)}-\cdots} \\
& -\frac{J}{A_{2}^{(n)} A_{1}^{(n)}} \sum_{\substack{k_{1}+k_{2}+k_{3}=m \\
k_{1}, k_{2}, k_{3} \geq 0}}\left(\begin{array}{c}
m \\
k_{1}, k_{2}, k_{3}
\end{array}\right)(-1)^{k_{1}} \mathfrak{T}_{2, k_{1}}^{\left(s_{2,0}^{(n)}, \cdots, s_{2,3}^{(n)}\right)} \mathfrak{T}_{1, k_{2}}^{\left(s_{1,0}^{(n)}, \cdots, s_{1,3}^{(n)}\right)},
\end{aligned}
$$

where $A=-D+E+G+H-3, B=12 D+12 G-4 J-12, \quad C=-E-2 G-H+4$, $F=-2 D-2 G-2 H+6, \quad I=4 D-E+2 G-H-J, \quad t_{1, m}^{(n)}$ is determined in Theorem 6.3.

Lemma 4.3 will be discussed in four cases.

\subsection{Case 1}

$B=C=D=0$.

Theorem 6.7. For $m \geq 0, n \geq 1$,

$$
\begin{aligned}
& \frac{1}{\left(A_{1}^{(n)}\right)^{5}} \sum_{\substack{k_{1}+\cdots+k_{5}=m \\
k_{1}, \cdots, k_{5} \geq 0}}\left(\begin{array}{c}
m \\
k_{1}, \cdots, k_{5}
\end{array}\right) \mathfrak{T}_{1, k_{1}}^{\left(s_{1,0}^{(n)}, \cdots, s_{1,3}^{(n)}\right)} \cdots \mathfrak{T}_{1, k_{5}}^{\left(s_{1,0}^{(n)}, \cdots, s_{1,3}^{(n)}\right)} \\
& =\frac{A}{A_{1}^{(5 n)}} 5^{m} \mathfrak{T}_{1, m}^{\left(s_{1,0}^{(5 n)}, \cdots, s_{1,3}^{(5 n)}\right)}+\frac{E}{A_{1}^{(4 n)} A_{1}^{n}} \sum_{k=0}^{m}\left(\begin{array}{c}
m \\
k
\end{array}\right) 4^{m-k} \mathfrak{T}_{1, m-k}^{\left(s_{1,0}^{(4 n)}, \cdots, s_{1,3}^{(4 n)}\right)} \mathfrak{T}_{1, k}^{\left(s_{1,0}^{(n)}, \cdots, s_{1,3}^{(n)}\right)} \\
& \quad+F\left(\frac{-1}{563}\right)^{n} \sum_{k=0}^{m}\left(\begin{array}{c}
m \\
k
\end{array}\right) \mathfrak{T}_{1, k}^{\left(s_{1,0}^{(n)}, \cdots, s_{1,3}^{(n)}\right)}+\frac{G}{A_{1}^{(n)}} \sum_{k=0}^{m}\left(\begin{array}{c}
m \\
k
\end{array}\right) t_{2, k}^{(n)} \mathfrak{T}_{1, m-k}^{\left(s_{1,0}^{(n)}, \cdots, s_{1,3}^{(n)}\right)} \\
& \quad+\frac{H}{A_{1}^{(3 n)} A_{1}^{(2 n)}} \sum_{k=0}^{m}\left(\begin{array}{c}
m \\
k
\end{array}\right) 3^{m-k} 2^{k} \mathfrak{T}_{1, m-k}^{\left(s_{1,0}^{(3 n)}, \cdots, s_{1,3}^{(3 n)}\right)} \mathfrak{T}_{1, k}^{\left(s_{1,0}^{(2 n)}, \cdots, s_{1,3}^{(2 n)}\right)}
\end{aligned}
$$




$$
\begin{aligned}
& +\frac{I}{A_{1}^{(3 n)}} \sum_{k=0}^{m}\left(\begin{array}{c}
m \\
k
\end{array}\right) 3^{k} \mathfrak{T}_{1, k}^{\left(s_{1,0}^{(3 n)}, \cdots, s_{1,3}^{(3 n)}\right)} t_{1, m-k}^{(n)} \\
& +\frac{J}{A_{2}^{(n)} A_{1}^{(2 n)}} \sum_{\substack{k_{1}+k_{2}+k_{3}=m \\
k_{1}, k_{2}, k_{3} \geq 0}}\left(\begin{array}{c}
m \\
k_{1}, k_{2}, k_{3}
\end{array}\right)(-1)^{k_{1}} \mathfrak{T}_{2, k_{1}}^{\left(s_{2}(n), \cdots, s_{2,3}^{(n)}\right)} 2^{k_{2}} \mathfrak{T}_{1, k_{2}}^{\left(s_{1,0}^{(2 n)}, \cdots, s_{1,3}^{(2 n)}\right)} \\
& +\frac{K}{A_{2}^{(n)}} \sum_{\substack{k_{1}+k_{2}+k_{3}=m \\
k_{1}, k_{2}, k_{3} \geq 0}}\left(\begin{array}{c}
m \\
k_{1}, k_{2}, k_{3}
\end{array}\right)(-1)^{k_{1}} \mathfrak{T}_{2, k_{1}}^{\left(s_{2,0}^{(n)}, \cdots, s_{2,3}^{(n)}\right)} t_{1, k_{2}}^{(n)} \\
& +\frac{L}{A_{1}^{(3 n)}\left(A_{1}^{(n)}\right)^{2}} \sum_{\substack{k_{1}+k_{2}+k_{3}=m \\
k_{1}, k_{2}, k_{3} \geq 0}}\left(\begin{array}{c}
m \\
k_{1}, k_{2}, k_{3}
\end{array}\right) 3^{k_{1}} \mathfrak{T}_{1, k_{1}}^{\left(s_{1,0}^{(3 n)}, \cdots, s_{1,3}^{(3 n)}\right)} \mathfrak{T}_{1, k_{2}}^{\left(s_{1,0}^{(n)}, \cdots, s_{1,3}^{(n)}\right)} \mathfrak{T}_{1, k_{3}}^{\left(s_{1,0}^{(n)}, \cdots, s_{1,3}^{(n)}\right)} \\
& +\frac{M}{A_{2}^{(n)}\left(A_{1}^{(n)}\right)^{2}} \sum_{\substack{k_{1}+k_{2}+k_{3}+k_{4}=m \\
k_{1}, k_{2}, k_{3}, k_{4} \geq 0}}\left(\begin{array}{c}
m \\
k_{1}, k_{2}, k_{3}, k_{4}
\end{array}\right)(-1)^{k_{1}} \mathfrak{T}_{2, k_{1}, \cdots, s_{2,3}}^{\left(s_{2,1}^{(n)}\right)} \mathfrak{T}_{1, k_{2}}^{\left(s_{1,0}^{(n)}, \cdots, s_{1,3}^{(n)}\right.} \mathfrak{T}_{1, k_{3}}^{\left(s_{1,0}^{(n)}, \cdots, s_{1,3}^{(n)}\right)} \\
& +\frac{N}{\left(A_{1}^{(2 n)}\right)^{2} A_{1}^{n}} \sum_{\substack{k_{1}+k_{2}+k_{3}=m \\
k_{1}, k_{2}, k_{3} \geq 0}}\left(\begin{array}{c}
m \\
k_{1}, k_{2}, k_{3}
\end{array}\right) 2^{k_{1}} \mathfrak{T}_{1, k_{1}}^{\left(s_{1,0}^{(2 n)}, \cdots, s_{1,3}^{(2 n)}\right)} 2^{k_{2}} \mathfrak{T}_{1, k_{2}}^{\left(s_{1,0}^{(2 n)}, \cdots, s_{1,3}^{(2 n)}\right)} \mathfrak{T}_{1, k_{3}}^{\left(s_{1,0}^{(n)}, \cdots, s_{1,3}^{(n)}\right)} \\
& +\frac{P}{A_{1}^{(n)}} \sum_{\substack{k_{1}+k_{2}+k_{3}=m \\
k_{1}, k_{2}, k_{3} \geq 0}}\left(\begin{array}{c}
m \\
k_{1}, k_{2}, k_{3}
\end{array}\right) t_{1, k_{1}}^{(n)} t_{1, k_{2}}^{(n)} \mathfrak{T}_{1, k_{3}}^{\left(s_{1,0}^{(n)}, \cdots, s_{1,3}^{(n)}\right)} \\
& +\frac{Q}{A_{1}^{(2 n)} A_{1}^{(n)}} \sum_{\substack{k_{1}+k_{2}+k_{3}=m \\
k_{1}, k_{2}, k_{3} \geq 0}}\left(\begin{array}{c}
m \\
k_{1}, k_{2}, k_{3}
\end{array}\right) 2^{k_{1}} \mathfrak{T}_{1, k_{1}}^{\left(s_{1,0}^{(2 n)}, \cdots, s_{1,3}^{(2 n)}\right)} t_{1, k_{2}}^{(n)} \mathfrak{T}_{1, k_{3}}^{\left(s_{1,0}^{(n)}, \cdots, s_{1,3}^{(n)}\right)} \\
& +\frac{R}{A_{1}^{(2 n)}\left(A_{1}^{(n)}\right)^{3}} \sum_{\substack{k_{1}+k_{2}+k_{3}+k_{4}=m \\
k_{1}, k_{2}, k_{3}, k_{4} \geq 0}}\left(\begin{array}{c}
m \\
k_{1}, k_{2}, k_{3}, k_{4}
\end{array}\right) 2^{k_{1}} \mathfrak{T}_{1, k_{1}}^{\left(s_{1,0}^{(2 n)}, \cdots, s_{1,3}^{(2 n)}\right)} \mathfrak{T}_{1, k_{2}}^{\left(s_{1,0}^{(n)}, \cdots, s_{1,3}^{(n)}\right)} \\
& \mathfrak{T}_{1, k_{3}}^{\left(s_{1,0}^{(n)}, \cdots, s_{1,3}^{(n)}\right)} \mathfrak{T}_{1, k_{4}}^{\left(s_{1,0}^{(n)}, \cdots, s_{1,3}^{(n)}\right)} \\
& +\frac{S}{\left(A_{1}^{(n)}\right)^{3}} \sum_{\substack{k_{1}+k_{2}+k_{3}+k_{4}=m \\
k_{1}, k_{2}, k_{3}, k_{4} \geq 0}}\left(\begin{array}{c}
m \\
k_{1}, k_{2}, k_{3}, k_{4}
\end{array}\right) t_{1, k_{1}}^{(n)} \mathfrak{T}_{1, k_{2}}^{\left(s_{1,0}^{(n)}, \cdots, s_{1,3}^{(n)}\right)} \mathfrak{T}_{1, k_{3}}^{\left(s_{1,0}^{(n)}, \cdots, s_{1,3}^{(n)}\right)} \mathfrak{T}_{1, k_{4}}^{\left(s_{1,0}^{(n)}, \cdots, s_{1,3}^{(n)}\right)},
\end{aligned}
$$

where

$$
\begin{aligned}
& A=I+2 L+2 N+P+2 Q+6 R+4 S-14, \\
& E=-I-2 L-N-Q-3 R-S+5, \\
& F=4 G+I+2 L+6 N+5 P+6 Q+18 R+16 S-50, \\
& H=-L-2 N-P-Q-4 R-3 S+10, \\
& J=-G-I-2 L-M-2 P-3 Q-6 R-7 S+20, \\
& K=-2 G-2 M-2 N-5 P-2 Q-6 R-12 S+30,
\end{aligned}
$$

$t_{1, m}^{(n)}$ and $t_{2, m}^{(n)}$ are determined in Theorem 6.3 and 6.6, respectively. 


\subsection{Case 2}

$B \neq 0, C=D=0$. Let

$$
\begin{aligned}
& \sum_{k=0}^{\infty} t_{3, k}^{(n)} \frac{x^{k}}{k !} \\
& =c_{1}^{n} c_{2}^{n} c_{3}^{n} e^{(\alpha+\beta+\gamma) x}\left(c_{1}^{n} c_{2}^{n} e^{(\alpha+\beta) x}+c_{2}^{n} c_{3}^{n} e^{(\beta+\gamma) x}+c_{3}^{n} c_{1}^{n} e^{(\gamma+\alpha) x}\right)+\cdots \\
& \quad+c_{2}^{n} c_{3}^{n} c_{4}^{n} e^{(\beta+\gamma+\delta) x}\left(c_{2}^{n} c_{3}^{n} e^{(\beta+\gamma) x}+c_{3}^{n} c_{4}^{n} e^{(\gamma+\delta) x}+c_{4}^{n} c_{2}^{n} e^{(\delta+\beta) x}\right) .
\end{aligned}
$$

Theorem 6.8. For $m \geq 0, n \geq 1$,

$$
\begin{aligned}
& B t_{3, m}^{(n)}=\frac{1}{\left(A_{1}^{(n)}\right)^{5}} \sum_{\substack{k_{1}+\cdots, k_{5}=m \\
k_{1}, \cdots, k_{5} \geq 0}}\left(\begin{array}{c}
m \\
k_{1}, \cdots, k_{5}
\end{array}\right) \mathfrak{T}_{1, k_{1}}^{\left(s_{1,0}^{(n)}, \cdots, s_{1,3}^{(n)}\right)} \cdots \mathfrak{T}_{1, k_{5}}^{\left(s_{1,0}^{(n)}, \cdots, s_{1,3}^{(n)}\right)} \\
& \quad-\frac{A}{A_{1}^{(5 n)}} 5^{m} \mathfrak{T}_{1, m}^{\left(s_{1,0}^{(5 n)}, \cdots, s_{1,3}^{(5 n)}\right)}-\cdots \\
& \quad-\frac{S}{\left(A_{1}^{(n)}\right)^{3}} \sum_{\substack{k_{1}+k_{2}+k_{3}+k_{4}=m \\
k_{1}, k_{2}, k_{3}, k_{4} \geq 0}}\left(\begin{array}{c}
m \\
k_{1}, k_{2}, k_{3}, k_{4}
\end{array}\right) t_{1, k_{1}}^{(n)} \mathfrak{T}_{1, k_{2}}^{\left(s_{1,0}^{(n)}, \cdots, s_{1,3}^{(n)}\right)} \mathfrak{T}_{1, k_{3}}^{\left(s_{1,0}^{n}, \cdots, s_{1,3}^{(n)}\right)} \mathfrak{T}_{1, k_{4}}^{\left(s_{1,0}^{(n)}, \cdots, s_{1,3}^{(n)}\right)},
\end{aligned}
$$

where

$$
\begin{aligned}
& A=-G-J-M+2 N-P-Q-3 S+6 \\
& B=-2 G-K-2 M-2 N-5 P-2 Q-6 R-12 S+30 \\
& E=G+J+M-N+2 P+2 Q+3 R+6 S-15 \\
& F=-3 G-J-3 K-7 M-12 P-3 Q-6 R-27 S+60 \\
& H=-L-2 N-P-Q-4 R-3 S+10 \\
& I=-G-J-2 L-M-2 P-3 Q-6 R-7 S+20
\end{aligned}
$$

$t_{1, m}^{(n)}$ and $t_{2, m}^{(n)}$ are determined in Theorem 6.3 and 6.6, respectively.

\subsection{Case 3}

$C \neq 0, B=D=0$. Let

$$
\begin{aligned}
& \sum_{k=0}^{\infty} t_{4, k}^{(n)} \frac{x^{k}}{k !} \\
& =c_{1}^{n} c_{2}^{n} c_{3}^{n} e^{(\alpha+\beta+\gamma) x}\left(c_{1}^{2 n} e^{2 \alpha x}+c_{2}^{2 n} e^{2 \beta x}+c_{3}^{2 n} e^{2 \gamma x}\right)+\cdots \\
& \quad+c_{2}^{n} c_{3}^{n} c_{4}^{n} e^{(\beta+\gamma+\delta) x}\left(c_{2}^{2 n} e^{2 \beta x}+c_{3}^{2 n} e^{2 \gamma x}+c_{4}^{2 n} e^{2 \delta x}\right) .
\end{aligned}
$$

Theorem 6.9. For $m \geq 0, n \geq 1$,

$$
C t_{4, m}^{(n)}=\frac{1}{\left(A_{1}^{(n)}\right)^{5}} \sum_{\substack{k_{1}+\cdots+k_{5}=m \\
k_{1}, \cdots, k_{5} \geq 0}}\left(\begin{array}{c}
m \\
k_{1}, \cdots, k_{5}
\end{array}\right) \mathfrak{T}_{1, k_{1}}^{\left(s_{1,0}^{(n)}, \cdots, s_{1,3}^{(n)}\right)} \cdots \mathfrak{T}_{1, k_{5}}^{\left(s_{1,0}^{(n)}, \cdots, s_{1,3}^{(n)}\right)}
$$




$$
\begin{aligned}
& -\frac{A}{A_{1}^{(5 n)}} 5^{m} \mathfrak{T}_{1, m}^{\left(s_{1,0}^{(5 n)}, \cdots, s_{1,3}^{(5 n)}\right)}-\cdots \\
& -\frac{S}{\left(A_{1}^{(n)}\right)^{3}} \sum_{\substack{k_{1}+k_{2}+k_{3}+k_{4}=m \\
k_{1}, k_{2}, k_{3}, k_{4} \geq 0}}\left(\begin{array}{c}
m \\
k_{1}, k_{2}, k_{3}, k_{4}
\end{array}\right) t_{1, k_{1}}^{(n)} \mathfrak{T}_{1, k_{2}}^{\left(s_{1,0}^{(n)}, \cdots, s_{1,3}^{(n)}\right)} \mathfrak{T}_{1, k_{3}}^{\left(s_{1,0}^{(n)}, \cdots, s_{1,3}^{(n)}\right)} \mathfrak{T}_{1, k_{4}}^{\left(s_{1,0}^{(n)}, \cdots, s_{1,3}^{(n)}\right)},
\end{aligned}
$$

where

$$
\begin{aligned}
& A=I+2 L+2 N+P+2 Q+6 R+4 S-14, \\
& C=-G-I-J-2 L-M-2 P-3 Q-6 R-7 S+20, \\
& E=-I-2 L-N-Q-3 R-S+5 \\
& F=3 G-J-M+6 N+3 P+3 Q+12 R+9 S-30, \\
& H=-L-2 N-P-Q-4 R-3 S+10 \\
& K=-2 G-2 M-2 N-5 P-2 Q-6 R-12 S+30,
\end{aligned}
$$

$t_{1, m}^{(n)}$ and $t_{2, m}^{(n)}$ are determined in Theorem 6.3 and 6.6, respectively.

\subsection{Case 4}

$D \neq 0, B=C=0$. Let

$$
\begin{aligned}
& \sum_{k=0}^{\infty} t_{5, k}^{(n)} \frac{x^{k}}{k !} \\
& =c_{1}^{n} c_{2}^{n} c_{3}^{n} e^{(\alpha+\beta+\gamma) x}\left(c_{1}^{n} e^{\alpha x}+c_{2}^{n} e^{\beta x}+c_{3}^{n} e^{\gamma x}\right)^{2}+\cdots \\
& \quad+c_{2}^{n} c_{3}^{n} c_{4}^{n} e^{(\beta+\gamma+\delta) x}\left(c_{2}^{n} e^{\beta x}+c_{3}^{n} e^{\gamma x}+c_{4}^{n} e^{\delta x}\right)^{2}
\end{aligned}
$$

Theorem 6.10. For $m \geq 0, n \geq 1$,

$$
\begin{aligned}
& D t_{5, m}^{(n)}=\frac{1}{\left(A_{1}^{(n)}\right)^{5}} \sum_{\substack{k_{1}+\cdots, k_{5}=m \\
k_{1}, \cdots, k_{5} \geq 0}}\left(\begin{array}{c}
m \\
k_{1}, \cdots, k_{5}
\end{array}\right) \mathfrak{T}_{1, k_{1}}^{\left(s_{1,0}^{(n)}, \cdots, s_{1,3}^{(n)}\right)} \cdots \mathfrak{T}_{1, k_{5}}^{\left(s_{1,0}^{(n)}, \cdots, s_{1,3}^{(n)}\right)} \\
& \quad-\frac{A}{A_{1}^{(5 n)}} 5^{m} \mathfrak{T}_{1, m}^{\left(s_{1,0}^{(5 n)}, \cdots, s_{1,3}^{(n)}\right)}-\cdots \\
& \quad-\frac{S}{\left(A_{1}^{(n)}\right)^{3}} \sum_{\substack{k_{1}+k_{2}+k_{3}+k_{4}=m \\
k_{1}, k_{2}, k_{3}, k_{4} \geq 0}}\left(\begin{array}{c}
m \\
k_{1}, k_{2}, k_{3}, k_{4}
\end{array}\right) t_{1, k_{1}}^{(n)} \mathfrak{T}_{1, k_{2}}^{\left(s_{1,0}^{(n)}, \cdots, s_{1,3}^{(n)}\right)} \mathfrak{T}_{1, k_{3}}^{\left(s_{1,0}^{(n)}, \cdots, s_{1,3}^{(n)}\right)} \mathfrak{T}_{1, k_{4}}^{\left(s_{1,0}^{(n)}, \cdots, s_{1,3}^{(n)}\right)},
\end{aligned}
$$

where

$$
\begin{aligned}
& A=I+2 L+2 N+P+2 Q+6 R+4 S-14, \\
& D=-G-I-J-2 L-M-2 P-3 Q-6 R-7 S+20, \\
& E=-I-2 L-N-Q-3 R-S+5 \\
& F=-3 G-6 I-7 J-7 M+6 N-12 L-9 P-15 Q-24 R-33 S+90, \\
& H=-L-2 N-P-Q-4 R-3 S+10 \\
& K=2 I+2 J-2 N+4 L-P+4 Q+6 R+2 S-10,
\end{aligned}
$$

$t_{1, m}^{(n)}$ and $t_{2, m}^{(n)}$ are determined in Theorem 6.3 and 6.6, respectively. 


\section{Some more interesting general expressions}

We shall give some more interesting general expressions.

Lemma 7.1. For $n \geq 1$, we have

$$
\begin{aligned}
& \left(c_{2} c_{3}+c_{3} c_{4}+c_{4} c_{2}\right) e^{\alpha x}+\left(c_{3} c_{4}+c_{4} c_{1}+c_{1} c_{3}\right) e^{\beta x} \\
& +\left(c_{1} c_{2}+c_{1} c_{4}+c_{4} c_{2}\right) e^{\gamma x}+\left(c_{1} c_{2}+c_{2} c_{3}+c_{1} c_{3}\right) e^{\delta x} \\
= & \frac{1}{563} \sum_{k=0}^{\infty} \mathfrak{T}_{k}^{(146,416,581,1080)} \frac{x^{k}}{k !} .
\end{aligned}
$$

\section{Theorem 7.2.}

$$
\begin{aligned}
& \left(c_{2} c_{3}+c_{3} c_{4}+c_{4} c_{2}\right)^{n} e^{\alpha x}+\left(c_{3} c_{4}+c_{4} c_{1}+c_{1} c_{3}\right)^{n} e^{\beta x} \\
& +\left(c_{1} c_{2}+c_{1} c_{4}+c_{4} c_{2}\right)^{n} e^{\gamma x}+\left(c_{1} c_{2}+c_{2} c_{3}+c_{1} c_{3}\right)^{n} e^{\delta x} \\
= & \frac{1}{A_{3}^{n}} \sum_{k=0}^{\infty} \mathfrak{T}_{3, k}^{\left(s_{3,0}^{n}, s_{3,1}^{n}, s_{3,2}^{n}, s_{3,3}^{n}\right)} \frac{x^{k}}{k !},
\end{aligned}
$$

where $s_{3,0}^{(n)}, s_{3,1}^{(n)}, s_{3,2}^{(n)}, s_{3,3}^{(n)}$ and $A_{3}^{(n)}$ satisfy the recurrence relation:

$$
\begin{aligned}
& s_{3,0}^{(n)}= \pm \operatorname{lcm}\left(b_{1}, b_{2}, b_{3}\right), \quad s_{3,1}^{(n)}=M s_{3,0}^{(n)}, \quad s_{3,2}^{(n)}=N s_{3,0}^{(n)}, \quad s_{3,3}^{(n)}=P s_{3,0}^{(n)}, \\
& A_{3}^{(n)}=A_{3}^{(n-1)} \frac{\left(-16 s_{3,3}^{(n)}+103 s_{3,2}^{(n)}-157 s_{3,1}^{(n)}-10 s_{3,0}^{(n)}\right)}{-8 s_{3,3}^{(n)}+10 s_{3,2}^{(n)}+7 s_{3,1}^{(n)}-6 s_{3,0}^{(n)}} .
\end{aligned}
$$

$b_{1}, b_{2}, b_{3}, M, N$ and $P$ are determined in the proof.

Proof. Similarly to the proof of Theorem 6.3, we consider the form

$$
h_{1}^{(n)} e^{\alpha x}+h_{2}^{(n)} e^{\beta x}+h_{3}^{(n)} e^{\gamma x}+h_{4}^{(n)} e^{\delta x}=\sum_{k=0}^{\infty} s_{3, k}^{(n)} \frac{x^{k}}{k !} .
$$

By $h_{1}^{(n)}=A_{3}^{(n)}\left(c_{2} c_{3}+c_{3} c_{4}+c_{4} c_{2}\right)^{n}$, we can obtain the following recurrence relation:

$$
\begin{aligned}
& A=-650 s_{3,0}^{(n-1)}+385 s_{3,1}^{(n-1)}+854 s_{3,2}^{(n-1)}-664 s_{3,3}^{(n-1)}, \\
& B=1862 s_{3,0}^{(n-1)}+231 s_{3,1}^{(n-1)}-1627 s_{3,2}^{(n-1)}+1178 s_{3,3}^{(n-1)}, \\
& C=-1100 s_{3,0}^{(n-1)}-2380 s_{3,1}^{(n-1)}-417 s_{3,2}^{(n-1)}+522 s_{3,3}^{(n-1)}, \\
& D=16 s_{3,0}^{(n-1)}-252 s_{3,1}^{(n-1)}-170 s_{3,2}^{(n-1)}+148 s_{3,3}^{(n-1)}, \\
& E=1198 s_{3,0}^{(n-1)}-1083 s_{3,1}^{(n-1)}-1906 s_{3,2}^{(n-1)}+1368 s_{3,3}^{(n-1)}, \\
& F=-2434 s_{3,0}^{(n-1)}+814 s_{3,1}^{(n-1)}+3473 s_{3,2}^{(n-1)}-1769 s_{3,3}^{(n-1)}, \\
& G=988 s_{3,0}^{(n-1)}+1935 s_{3,1}^{(n-1)}-757 s_{3,2}^{(n-1)}-933 s_{3,3}^{(n-1)}, \\
& H=-518 s_{3,0}^{(n-1)}+801 s_{3,1}^{(n-1)}+920 s_{3,2}^{(n-1)}-834 s_{3,3}^{(n-1)}, \\
& I=268 s_{3,0}^{(n-1)}+186 s_{3,1}^{(n-1)}-132 s_{3,2}^{(n-1)}-16 s_{3,3}^{(n-1)},
\end{aligned}
$$




$$
\begin{gathered}
J=-1303 s_{3,0}^{(n-1)}-1690 s_{3,1}^{(n-1)}+146 s_{3,2}^{(n-1)}+666 s_{3,3}^{(n-1)}, \\
K=-3980 s_{3,0}^{(n-1)}-3273 s_{3,1}^{(n-1)}+1638 s_{3,2}^{n-1}+620 s_{3,3}^{(n-1)}, \\
L=1012 s_{3,0}^{(n-1)}-869 s_{3,1}^{(n-1)}-1490 s_{3,2}^{(n-1)}+1116 s_{3,3}^{(n-1)}, \\
M=\frac{(L A-D I)(F A-B E)-(H A-D E)(J A-B I)}{(G A-C E)(J A-B I)-(K A-C I)(F A-B E)}, \\
N=\frac{M(G A-C E)+(H A-D E)}{B E-F A}, \\
P=-\frac{1}{A}(B N+C M+D), \\
M=\frac{a_{1}}{b_{1}}, \quad N=\frac{a_{2}}{b_{2}}, \quad P=\frac{a_{3}}{b_{3}}, \quad \operatorname{gcd}\left(a_{i}, b_{i}\right)=1, \\
s_{3,0}^{(n)}= \pm \operatorname{lcm}\left(b_{1}, b_{2}, b_{3}\right), \quad s_{3,1}^{(n)}=M s_{3,0}^{(n)}, \quad s_{3,2}^{(n)}=N s_{3,0}^{(n)}, \quad s_{3,3}^{(n)}=P s_{3,0}^{(n)}, \\
A_{3}^{(n)}=A_{3}^{(n-1)} \frac{\left(-16 s_{3,3}^{(n)}+103 s_{3,2}^{(n)}-157 s_{3,1}^{(n)}-10 s_{3,0}^{(n)}\right)}{-8 s_{3,3}^{(n)}+10 s_{3,2}^{(n)}+7 s_{3,1}^{(n)}-6 s_{3,0}^{(n)}} .
\end{gathered}
$$

We choose the value of $s_{3,0}^{(n)}$ such that for some $k_{0}, \forall k \geq k_{0}, \mathfrak{T}_{3, k}^{\left(s_{3,0}^{(n)}, s_{3,1}^{(n)}, s_{3,2}^{(n)}, s_{3,3}^{(n)}\right)}$ is positive.

\section{Acknowledgements}

The authors thank the anonymous referees for careful reading of the manuscript and useful suggestions.

\section{References}

[1] Agoh, T., \& Dilcher, K. (2007). Convolution identities and lacunary recurrences for Bernoulli numbers, J. Number Theory, 124, 105-122.

[2] Agoh, T., \& Dilcher, K. (2009). Higher-order recurrences for Bernoulli numbers, J. Number Theory, 129, 1837-1847.

[3] Agoh, T., \& Dilcher, K. (2014). Higher-order convolutions for Bernoulli and Euler polynomials, J. Math. Anal. Appl., 419, 1235-1247.

[4] Kiliç, E. (2008). Formulas for sums of generalized order- $k$ Fibonacci type sequences by matrix methods, Ars Comb., 86, 395-402.

[5] Komatsu, T. (2015). Higher-order convolution identities for Cauchy numbers of the second kind, Proc. Jangjeon Math. Soc., 18, 369-383.

[6] Komatsu, T. (2016). Higher-order convolution identities for Cauchy numbers, Tokyo J. Math., 39, 225-239. 
[7] Komatsu, T. (2018). Convolution identities for Tribonacci numbers, Ars Combin., 136, 199-210.

[8] Komatsu, T., \& Li, R. (2019). Convolution identities for Tribonacci numbers with symmetric formulae, Math. Rep. (Bucur.), 21, 27-47.

[9] Komatsu, T., Masakova, Z., \& Pelantova, E. (2014). Higher-order identities for Fibonacci numbers, Fibonacci Quart., 52 (5), 150-163.

[10] Komatsu, T. \& Simsek, Y. (2016). Third and higher order convolution identities for Cauchy numbers, Filomat, 30, 1053-1060.

[11] Lin, P. Y. (1991). De Moivre-type identities for the Tetrabonacci numbers, Applications of Fibonacci numbers, 4 (Winston-Salem, NC, 1990), 215-218, Kluwer Acad. Publ., Dordrecht.

[12] Sloane, N. J. A. (2019). The On-Line Encyclopedia of Integer Sequences, Available online at: http://oeis.org.

[13] Waddill, M. E. (1992). The Tetranacci sequence and generalizations, Fibonacci Quart., 30, 9-20.

[14] Waddill, M. E. (1992). Some properties of the Tetranacci sequence modulo $m$, Fibonacci Quart., 30, 232-238.

[15] Young, P. T. (2003). On lacunary recurrences, Fibonacci Quart., 41, 41-47. 